\title{
The evolutionary status of Cataclysmic Variables: eclipse modelling of 15 systems
}

\author{
M. McAllister ${ }^{\oplus},{ }^{1}$ S. P. Littlefair ${ }^{\oplus},{ }^{\star}{ }^{\star}$ S. G. Parsons ${ }^{\oplus},{ }^{1}$ V. S. Dhillon, ${ }^{1,2}$ T. R. Marsh, ${ }^{3}$ \\ B. T. Gänsicke ${ }^{\oplus},{ }^{3}$ E. Breedt ${ }^{\oplus},{ }^{4}$ C. Copperwheat, ${ }^{5}$ M. J. Green ${ }^{\odot},{ }^{3}$ C. Knigge, ${ }^{6}$ \\ D. I. Sahman, ${ }^{1}$ Martin J. Dyer ${ }^{\oplus},{ }^{1}$ P. Kerry, ${ }^{1}$ R. P. Ashley, ${ }^{3}$ P. Irawati ${ }^{7}$ and \\ S. Rattanasoon ${ }^{7}$ \\ ${ }^{1}$ Department of Physics and Astronomy, University of Sheffield, Sheffield S3 7RH, UK \\ ${ }^{2}$ Instituto de Astrofísica de Canarias (IAC), E-38200 La Laguna, Tenerife, Spain \\ ${ }^{3}$ Department of Physics, University of Warwick, Coventry CV4 7AL, UK \\ ${ }^{4}$ Institute of Astronomy, University of Cambridge, Madingley Road, Cambridge CB3 OHA, UK \\ ${ }^{5}$ Astrophysics Research Institute, Liverpool John Moores University, IC2, Liverpool Science Park L3 5RF, UK \\ ${ }^{6}$ School of Physics \& Astronomy, University of Southampton, Southampton SO17 1BJ, UK \\ ${ }^{7}$ National Astronomical Research Institute of Thailand, 191 Siriphanich Bldg., Huay Kaew Road, Chiang Mai 50200, Thailand
}

Accepted 2019 April 1. in original form 2018 December 14

\begin{abstract}
We present measurements of the component masses in 15 Cataclysmic Variables (CVs) 6 new estimates and 9 improved estimates. We provide new calibrations of the relationship between superhump period excess and mass ratio, and use this relation to estimate donor star masses for 225 superhumping CVs. With an increased sample of donor masses we revisit the implications for $\mathrm{CV}$ evolution. We confirm the high mass of white dwarfs in CVs, but find no trend in white dwarf mass with orbital period. We argue for a revision in the location of the orbital period minimum of CVs to $79.6 \pm 0.2 \mathrm{~min}$, significantly shorter than previous estimates. We find that $\mathrm{CV}$ donors below the gap have an intrinsic scatter of only $0.005 \mathrm{R}_{\odot}$ around a common evolutionary track, implying a correspondingly small variation in angular momentum loss (AML) rates. In contrast to prior studies, we find that standard CV evolutionary tracks - without additional AML - are a reasonable fit to the donor masses just below the period gap, but that they do not reproduce the observed period minimum, or fit the donor radii below $0.1 \mathrm{M}_{\odot}$.
\end{abstract}

Key words: binaries: close-binaries: eclipsing-stars: dwarf novae-stars: evolutionnovae, cataclysmic variables - white dwarfs.

\section{INTRODUCTION}

Cataclysmic Variables (CVs) are close binary stars in which a white dwarf is accreting material from a low-mass donor star. Without angular momentum loss (AML) from the system, mass transfer could not be sustained; thus it is the AML that drives the secular evolution of CVs. The currently accepted picture of CV evolution is that CVs evolve from long to short periods under the influence of AML caused by magnetic braking. A reduction in AML due to magnetic braking is thought to arise when the donor becomes fully convective. This causes the CV to become detached, and is the cause of the dearth of $\mathrm{CVs}$ in the $2-3 \mathrm{~h}$ orbital period range; the $\mathrm{CV}$ period gap. When the $\mathrm{CV}$ resumes mass transfer, AML is driven by

^E-mail: s.littlefair@shef.ac.uk gravitational radiation and the mass transfer rate is lower. The $\mathrm{CV}$ evolves slowly through a period minimum, which arises because the thermal time-scale of the donor becomes comparable to the mass-loss time-scale, and the donor begins to expand in response to mass-loss, which leads to a widening of the orbit.

This long-standing picture has survived for over $35 \mathrm{yr}$ (Rappaport, Joss \& Webbink 1982; Rappaport, Verbunt \& Joss 1983) despite the fact that it struggles to explain the observed value of the period minimum (Gänsicke et al. 2009), the scarcity of known post-period-minimum systems (Hernández Santisteban et al. 2018) and the average high white dwarf mass in CVs (Zorotovic, Schreiber \& Gänsicke 2011). Modifications to the standard model exist that can potentially explain some of these issues. The orbital period minimum problem can be solved with an additional source of AML for short-period systems (Patterson 1998; Knigge, Baraffe \& Patterson 2011), and AML in nova outbursts may cause CVs with 
Table 1. Ephemerides for the CVs modelled in this paper. $T_{0}$ is the mid-eclipse time of cycle $0, P_{\text {orb }}$ is the orbital period, while $N_{\text {ecl }}$ is the total number of eclipses obtained.

\begin{tabular}{|c|c|c|c|c|c|c|c|}
\hline Object & $\begin{array}{c}\text { Right } \\
\text { ascension }\end{array}$ & Declination & $\begin{array}{l}\text { Out-of eclipse Mag. } \\
\qquad\left(g^{\prime}\right)\end{array}$ & $\begin{array}{c}T_{0} \\
\text { (MJD) }\end{array}$ & $\begin{array}{l}P_{\text {orb }} \\
\text { (d) }\end{array}$ & $N_{\text {ecl }}$ & $\begin{array}{l}\text { Add. Ecl. } \\
\text { Times }\end{array}$ \\
\hline CTCV J1300-3052 & 130029.05 & -305257.1 & 18.6 & $54262.099166(18)^{a}$ & $0.0889406998(17)$ & 4 & 1 \\
\hline DV UMa & 094636.65 & +444645.1 & 18.7 & $52782.973948(10)^{a}$ & $0.0858526308(7)$ & 4 & $2,3,4$ \\
\hline SDSS J115207.00+404947.8 & 115207.01 & +404948.0 & 19.5 & $55204.101279(6)^{a}$ & $0.0677497026(3)$ & 7 & 5 \\
\hline SDSS J150137.22+550123.4 & 150137.24 & +550123.5 & 19.0 & $56178.870444(8)^{a}$ & $0.05684126603(21)$ & 12 & - \\
\hline CSS080623 J140454-102702 & 140453.97 & -102702.3 & 19.5 & $55329.234631(13)^{a}$ & $0.059578971(3)$ & 10 & - \\
\hline CSS110113 J043112-031452 & 043112.45 & -031451.6 & 19.5 & $55942.014642(15)^{a}$ & $0.0660508707(18)$ & 12 & - \\
\hline GY Cnc & 090950.55 & +184947.5 & 16.7 & $55938.263734(22)^{b}$ & $0.175442399(6)$ & 12 & - \\
\hline IY UMa & 104356.73 & +580731.9 & 17.1 & $56746.6395010(9)^{a}$ & $0.07390892818(21)$ & 10 & 8 \\
\hline OY Car & 100622.07 & -701404.6 & 15.6 & $55353.996477(3)^{a}$ & $0.06312092545(24)$ & 7 & - \\
\hline SDSS J090103.94+480911.0 & 090103.94 & +480911.0 & 19.5 & $55942.116358(8)^{a}$ & $0.0778805321(5)$ & 10 & 9 \\
\hline SDSS J100658.40+233724.4 & 100658.42 & +233724.6 & 18.6 & $56682.72973(5)^{a}$ & $0.185913107(13)$ & 11 & 7,10 \\
\hline SSS130413 J094551-194402 & 094551.00 & -194400.8 & 16.7 & $56683.673971(12)^{a}$ & $0.0657692903(12)$ & 17 & 6 \\
\hline SSS100615 J200331-284941 & 200331.27 & -284941.3 & 19.6 & $56873.023625(5)^{a}$ & $0.0587045(4)$ & 3 & - \\
\hline V713 Cep & 204638.70 & +603802.8 & 18.5 & $56176.936402(7)^{a}$ & $0.0854185080(12)$ & 15 & 11 \\
\hline Z Cha & 080727.75 & -763200.7 & 15.6 & $53498.011471(4)^{a}$ & $0.0744992631(3)$ & 14 & - \\
\hline
\end{tabular}

Note: References for additional eclipse times: (1) Tappert, Augusteijn \& Maza (2004), (2) Howell et al. (1988), (3) Patterson et al. (2000), (4) Nogami et al. (2001), (5) Southworth et al. (2010), (6) Thorstensen, Alper \& Weil (2016), (7) Woudt (private communication), (8) Coppejans (private communication), (9) Dillon et al. (2008), (10) Southworth et al. (2007), and (11) Bours (private communication).

${ }^{a}$ Heliocentric times in HMJD(UTC).

${ }^{b}$ Barycentric times in BMJD(TDB).

low-mass white dwarfs to be unstable, explaining the high average white dwarf mass (Nelemans et al. 2016; Schreiber, Zorotovic \& Wijnen 2016). However, it remains to be seen if those modifications can correctly describe the observed properties of known CVs. In particular, the mass and radius of the donor star is a sensitive probe of the secular evolution. This is because the radius of the donor star in a $\mathrm{CV}$ can be inflated from the main-sequence value, by some amount that depends upon the mass-loss history of the donor. In particular, the donor radius is more likely to track the long-term average mass-loss rate than other physical properties of the $\mathrm{CV}$ such as the accretion light or the effective temperature of the accreting white dwarf (see Knigge et al. 2011, and references within).

One of the best methods of measuring donor masses and radii is to model the primary eclipse. During primary eclipse, the white dwarf and accretion disc are occulted, along with the bright-spot, located where the accretion stream impacts the outer rim of the disc. The path of the gas stream is determined by the mass ratio, and so the detailed shape of the primary eclipse contains enough information to derive extremely precise masses that are consistent with conventional spectroscopic methods (see Tulloch, RodríguezGil \& Dhillon 2009; Copperwheat et al. 2010; Savoury et al. 2012, for example). The photometric method has the advantage that it does not rely on detection of the light from the donor star, which is often invisible given the much brighter white dwarf and accretion disc, particularly for $\mathrm{CVs}$ with shorter orbital periods. It does however require high-quality light curves of the eclipses, which occur on time-scales of minutes. With this in mind, our group has been acquiring high-quality light curves of eclipsing CVs with the hightime-resolution instruments ULTRACAM (Dhillon et al. 2007) and ULTRASPEC (Dhillon et al. 2014). Here we present the analysis of 15 systems, and review the evolutionary status of CV systems in light of the results.

\subsection{Systems selected for eclipse modelling}

The 15 systems modelled in this paper are listed in Table 1. CTCV 1300, DV UMa, SDSS 1152, SDSS 1501 have existing mass determination from eclipse modelling of ULTRACAM data
(Savoury et al. 2011), whilst Z Cha, OY Car, IY UMa, GY Cnc, and SDSS 1006 have existing mass determinations in the literature from various methods (Wood et al. 1986; Wade \& Horne 1988; Wood \& Horne 1990; Thorstensen 2000; Steeghs et al. 2003; Southworth et al. 2009; Copperwheat et al. 2012). The existing mass determinations have large associated errors, and we re-analyse them here in the light of new data, and an updated modelling approach (see McAllister et al. 2017a, for details). The remaining 6 systems have no existing donor mass estimates, and were chosen from the eclipsing CVs observed with ULTRACAM/ULTRASPEC to date; the primary reason for their selection was an eclipse shape suitable for modelling, with visible white dwarf and bright-spot eclipses.

\section{OBSERVATIONS AND DATA REDUCTION}

The observations in this paper span a range of dates from 2003 May to 2017 Feb. All data were taken with the triple-band fast camera ULTRACAM, or the single-band fast camera ULTRASPEC. ULTRACAM data were taken on three telescopes; the 4.2-m William Herschel Telescope (WHT) situated at the Roque de los Muchachos Observatory on La Palma, Spain, the 8.2-m Very Large Telescope (VLT) at Paranal, Chile, and the 3.5-m New Technology Telescope (NTT) located at La Silla, Chile. All ULTRASPEC data were taken using the 2.4-m Thai National Telescope (TNT), located on Doi Inthanon in Thailand. All observations were obtained using the Sloan Digital Sky Survey (SDSS) filter set, with the exception of some of the ULTRASPEC observations, which use the KG5 filter. This filter is described in detail in Hardy et al. (2017); it is a broad-band filter encompassing the SDSS $u^{\prime}, g^{\prime}$, and $r^{\prime}$ passbands. For a full journal of observations, see Table C1 (Supporting Information).

Data reduction was carried out using the ULTRACAM pipeline reduction software (see Dhillon et al. 2007). One or more nearby, photometrically stable comparison stars were used to correct for transparency variations during observations. If the comparison stars have tabulated SDSS magnitudes, we used these to transform the photometry into the $u^{\prime} g^{\prime} r^{\prime} i^{\prime} z^{\prime}$ standard system (Smith et al. 2002), 
otherwise observations of standard stars from the nearest photometric night were used. Photometry was corrected for extinction using the median extinction coefficients for each observatory, as derived from long duration time-series taken with ULTRACAM and ULTRASPEC.

\section{METHODS}

\subsection{Orbital ephemerides}

Updated orbital ephemerides for the CVs in this paper were calculated, and are shown in Table 1. Mid-eclipse times were determined by averaging the time of white dwarf ingress and egress, as determined by locating the minima and maxima of a smoothed light-curve derivative. Mid-eclipse times were corrected to the Solar system Heliocentre or Barycentre using ASTROPY (The Astropy Collaboration 2018). The correction used was decided upon a system-to-system basis, and depended on previous mid-eclipse times and ephemerides in the literature. Heliocentric times are recorded in Coordinated Universal Time (UTC), Barycentric times in Barycentric Dynamical Time (TDB). Mid-eclipse times for each individual eclipse observed are presented in Table C1 (Supporting Information).

\subsection{Eclipse light-curve modelling}

The model used to fit the eclipse light curve is described by Savoury et al. (2011). The important assumptions in the model are that the bright-spot lies on the ballistic trajectory from the donor, the white dwarf follows a theoretical mass-radius relation and that the white dwarf is unobscured. The model has recently received two major improvements, as outlined in McAllister et al. (2017a). The model now has the ability to fit multiple light curves simultaneously whilst sharing parameters that do not change; such as the mass ratio $q$, the white dwarf eclipse width $\Delta \Phi$ and the white dwarf radius, scaled by the binary separation $R_{1} / a$. In addition, the model now has a statistical treatment of flickering using Gaussian Processes (GPs) that makes the uncertainty estimates for these parameter robust in the presence of flickering. For each system we either fit all the individual eclipses, or averaged several eclipses in the same filter. Averaging eclipses can ease convergence of the model, by reducing the number of free parameters, but it is not suitable when the light-curve features change between eclipses, for example due to a changing accretion disc radius.

Eclipse averaging was used for six systems: CSS080623, CSS110113, DV UMa, SDSS 0901, SDSS 1152, and SSS100615. All systems have multiple eclipse light curves observed close together in time (e.g. during the same observing run), and contain only low-amplitude flickering. When selecting eclipses for the construction of each average eclipse, great care was taken to exclude any eclipses with differing disc radius/flux and/or brightspot shape/flux changes. First, only eclipses obtained during the same observing run were considered for each average eclipse. Secondly, before averaging, all eclipses were phase-folded and overlaid, with any differing eclipses removed from consideration. An average eclipse was created for each available wavelength band, typically $u^{\prime} g^{\prime} r^{\prime}$ or $u^{\prime} g^{\prime} i^{\prime}$. As both CSS080623 and SDSS 0901 have multiple eclipses from two separate observing runs, two average eclipses in each wavelength band were created. For the remaining nine systems, we did not average light curves prior to fitting.

In general, the majority of eclipses showing a clear brightspot ingress feature were selected for modelling. However, for systems with many high-signal-to-noise eclipses containing very clear bright-spot eclipse features (e.g. OY Car and Z Cha), only six were selected. In these cases, the inclusion of additional eclipses had an insignificant effect on the system parameter values and errors, and did not justify the resulting increased model complexity and computational time. This approach was also taken with SSS130413 and V713 Cep, two systems with moderately clear bright-spot features.

\section{RESULTS}

\subsection{Simultaneous eclipse light-curve modelling}

For each of the 15, the chosen eclipses were fit with the CV eclipse model, with GPs used to model the flickering component. The binary model contains two possible versions of the bright-spot (see Savoury et al. 2011, for details). A more complex bright-spot model was used for all but three systems (SDSS 1501, SSS100615, V713 Cep). The simple bright-spot was used in these three systems due to each containing a weak bright-spot component in their eclipse light curves. The typical phase range of the eclipse light curves modelled was -0.10 to 0.15 ; however, an extended phase range was used for a number of systems. The phase range was increased for systems with a prominent bright-spot (e.g. CTCV 1300, GY Cnc, SDSS 1006) in addition to SDSS 1501 (tenuous bright-spot component) and V713 Cep (combination of heavy flickering post-eclipse and significant disc contribution).

Posterior probability distributions of all parameters in the binary model were estimated using a Markov Chain Monte Carlo (MCMC) approach. The full results of all eclipse fits are shown in Figs A1A15 (Supporting Information). Fig. 1 shows an example $g^{\prime}$-band eclipse light-curve fit for each system. In addition to the most probable fit of the eclipse model (blue line), a blue band is plotted that covers $1 \sigma$ from the mean of a random sample (size 1000) of the MCMC chain. The grey points represent the actual eclipse light curves, while the black points are the result of subtracting the GP's posterior mean (itself shown, $\pm 1 \sigma$, by the red band covering the residuals below each plot). Also plotted are the separate components of the eclipse model: white dwarf (purple), bright-spot (red), accretion disc (yellow), and donor (green).

\subsection{System parameters}

Once the parameters of the binary model are estimated, the system parameters can be found. A full discussion can be found in McAllister et al. (2017a). In brief, this involves an iterative procedure where the white dwarf spectral energy distribution (SED) - measured from the eclipse depth of the white dwarf - is fit by white dwarf atmosphere models (Bergeron, Wesemael \& Beauchamp 1995). This yields estimates of the white dwarf temperature $T_{1}$ and distance $d$. The values of $q, \Delta \Phi$ and $R_{1} / a$ from the binary model, combined with Kepler's third law and a temperature-corrected mass-radius relationship for the white dwarf, are used to calculate the posterior probability distribution functions (PDFs) of the system parameters:

(i) mass ratio, $q$;

(ii) white dwarf mass, $M_{1}$;

(iii) white dwarf radius, $R_{1}$;

(iv) white dwarf $\log g$;

(v) donor mass, $M_{2}$;

(vi) donor radius, $R_{2}$;

(vii) binary separation, $a$;

(viii) inclination, $i$. 

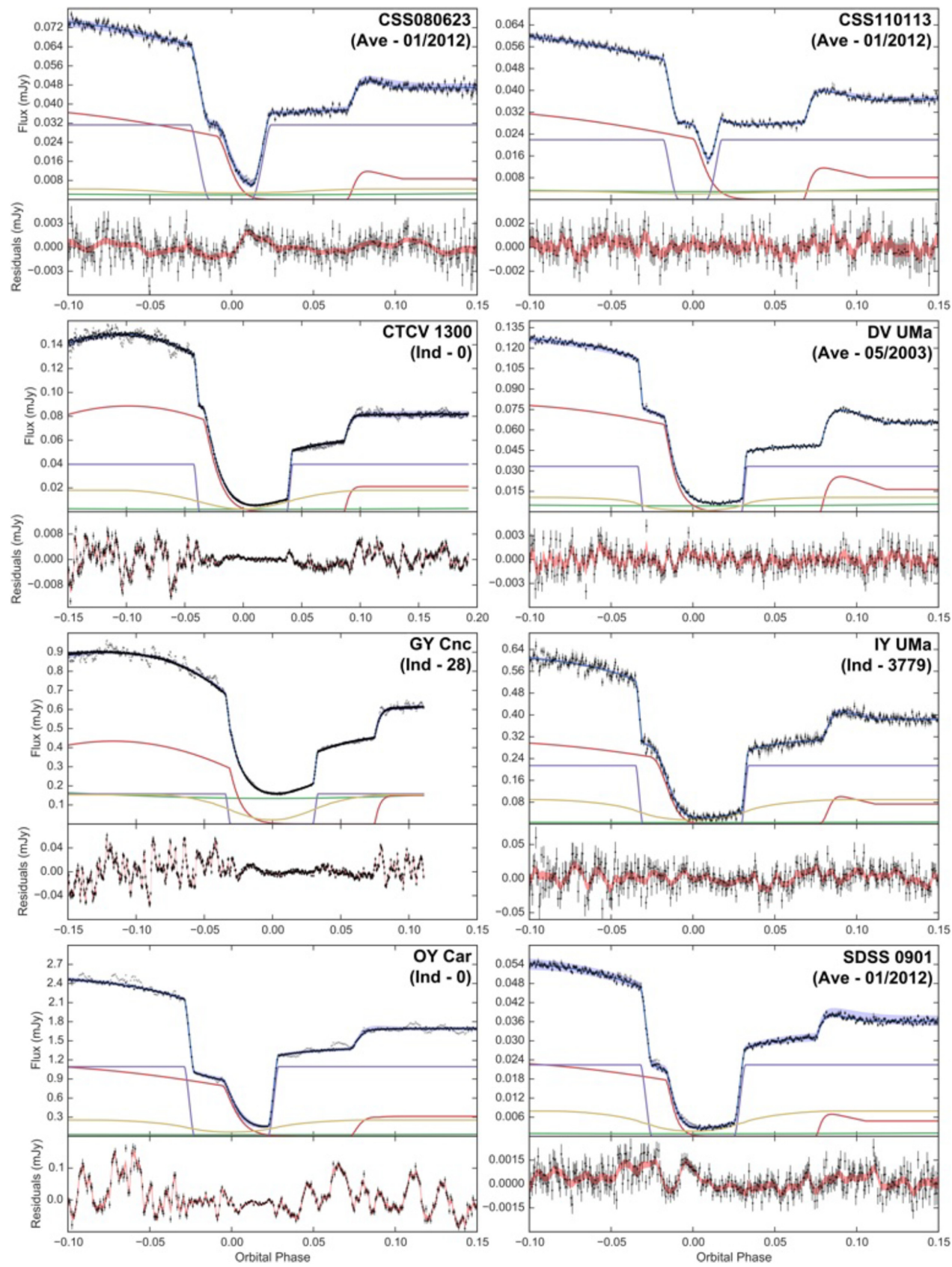

Figure 1. Eclipse model fits to $g$-band light curves of $15 \mathrm{CVs}$. The light curves are shown in grey points; see Section 4.1 for full details of what is plotted. The system name is displayed in the top-right corner of each plot, along with whether the eclipse is an individual (Ind) or average (Ave) eclipse. For individual eclipses the cycle number is shown, while for average eclipses the month and year of the eclipses are shown. See Appendix B for a complete set of eclipse plots.

System parameter values (see Table 2) were then obtained from the peak of each posterior PDF, with errors from the 67 per cent confidence level. The results of the white dwarf SED fits are shown in Fig. 2, and the resulting $T_{1}$ and $d$ values for each system are also displayed in Table 2. Note that the white dwarf flux fitting was not carried out for either IY UMa or SDSS 1006, due to the lack of $u^{\prime}$-band eclipses in their eclipse model fits. Thankfully, precise measurements of $T_{1}$ for both IY $\mathrm{UMa}^{1}(18000 \pm 1000 \mathrm{~K})$

${ }^{1}$ IY UMa entered outburst between the observations of Pala et al. (2017) and this work, so this $T_{1}$ measurement may be slightly lower than $T_{1}$ of the white dwarf in the eclipse light curves. and SDSS $1006(16000 \pm 1000 \mathrm{~K})$ from spectral fitting are given in Pala et al. (2017).

As a sanity check on our white dwarf atmosphere fitting, we can compare the derived distances with the parallaxes found in Gaia DR2 (Lindegren et al. 2018). We naively converted our distances to parallaxes, and compared to the parallaxes in Gaia DR2. The results are perfectly consistent with Gaussian statistics; the parallaxes of all but 4 out of $15 \mathrm{CVs}$ agree within 1 standard deviation, whilst the most discrepant $\mathrm{CV}$ (GY Cnc) has a $2 \sigma$ discrepancy between our derived distance and the Gaia DR2 parallax. This gives us confidence on our distance estimates and also their uncertainties. 

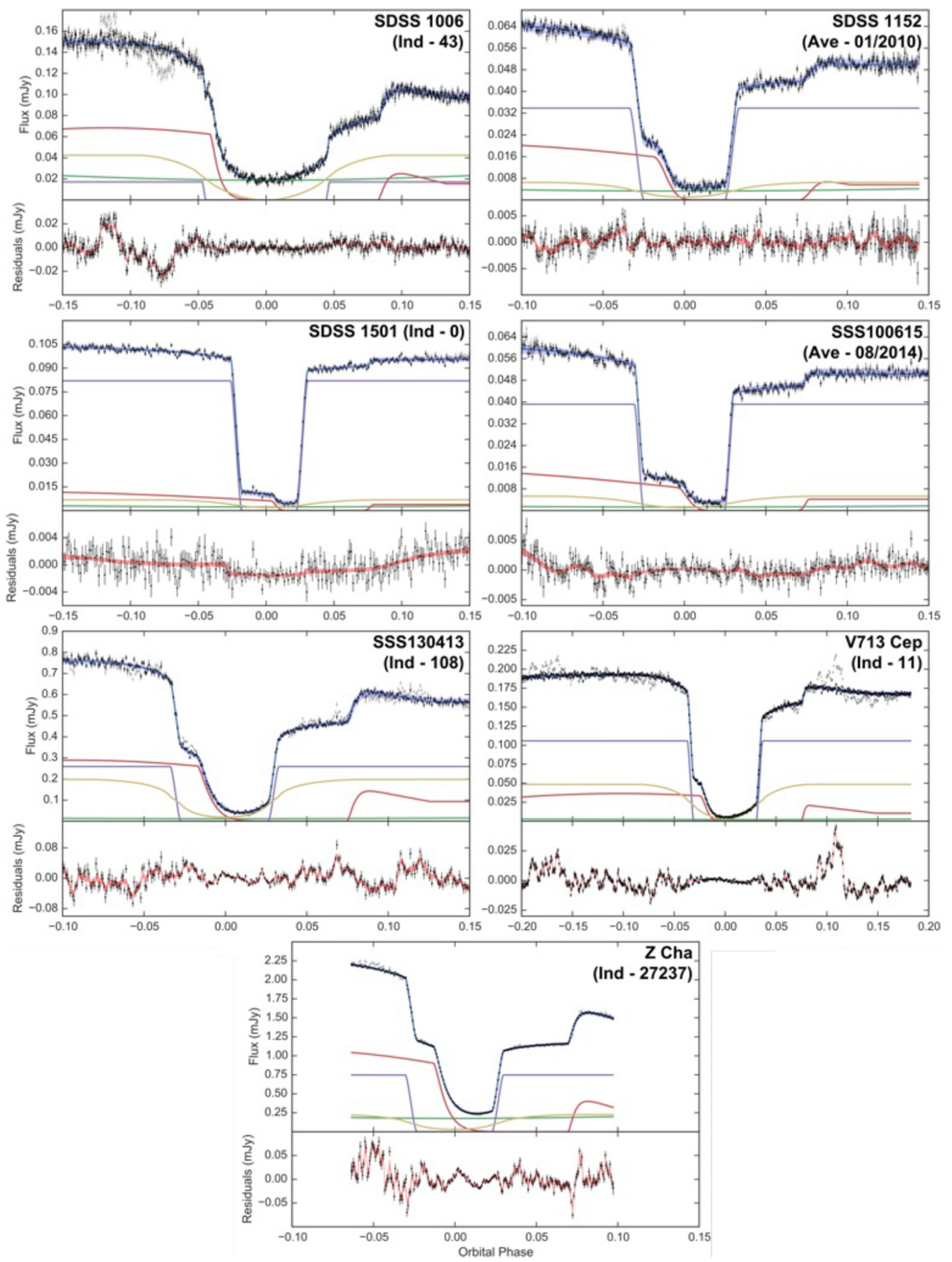

Figure 1. - continued

In Section 5, we discuss the implications of the measured system parameters for $\mathrm{CV}$ evolution. However, before then, we discuss some remarkable aspects of the data for two individual systems.

\subsection{White dwarf flux and orbital period variations in SDSS 1501}

The eclipses of SDSS 1501 are white dwarf dominated, but some show faint bright-spot features. There are a total of 15 available ULTRACAM eclipses of SDSS 1501, obtained during observing runs in 2004 (one eclipse), 2006 (eight), 2010 (two), and 2012 (one) [see Table C1 (Supporting Information) for further details]. However, only the single eclipses from 2004 and 2012 show signs of a bright-spot eclipse, so both ${ }^{2}$ were chosen for simultaneous eclipse modelling described above.

It became apparent that there was an appreciable increase in white dwarf flux across all three $\left(u^{\prime} g^{\prime} r^{\prime}\right)$ bands between the 2004 and 2012 eclipses. For this reason, model atmosphere fitting to the white dwarf fluxes was carried out separately for each eclipse, as shown in Fig. 2. The resulting $d$ and $T_{1}$ for each eclipse are shown in Table 2. The white dwarf in 2012 appears marginally hotter, but note the $1.6 \sigma$ discrepancy in $d$, which should of course remain constant. Both distances are formally consistent with a formal inference of the

${ }^{2}$ Each ULTRACAM eclipse is in three bands $\left(u^{\prime} g^{\prime} r^{\prime}\right)$, giving six individual eclipses for modelling. 
Table 2. System parameters for the 15 eclipsing systems analysed in this paper.

\begin{tabular}{|c|c|c|c|c|}
\hline Parameter & CSS080623 & CSS110113 & CTCV 1300 & DV UMa \\
\hline$q$ & $0.114 \pm 0.005$ & $0.105 \pm 0.006$ & $0.233 \pm 0.004$ & $0.172_{-0.007}^{+0.002}$ \\
\hline$M_{1}\left(\mathrm{M}_{\odot}\right)$ & $0.710 \pm 0.019$ & $1.00_{-0.01}^{+0.04}$ & $0.717 \pm 0.017$ & $1.09 \pm 0.03$ \\
\hline$R_{1}\left(\mathrm{R}_{\odot}\right)$ & $0.0117_{-0.0004}^{+0.0001}$ & $0.0080 \pm 0.0003$ & $0.01133 \pm 0.00021$ & $0.0072 \pm 0.0004$ \\
\hline$M_{2}\left(\mathrm{M}_{\odot}\right)$ & $0.081 \pm 0.005$ & $0.105 \pm 0.007$ & $0.166_{-0.003}^{+0.006}$ & $0.187_{-0.012}^{+0.003}$ \\
\hline$R_{2}\left(\mathrm{R}_{\odot}\right)$ & $0.1275 \pm 0.0024$ & $0.149 \pm 0.003$ & $0.2111_{-0.0014}^{+0.0025}$ & $0.215_{-0.005}^{+0.001}$ \\
\hline$a\left(\mathrm{R}_{\odot}\right)$ & $0.593 \pm 0.005$ & $0.711_{-0.003}^{+0.009}$ & $0.805 \pm 0.007$ & $0.889_{-0.012}^{+0.006}$ \\
\hline$K_{1}\left(\mathrm{~km} \mathrm{~s}^{-1}\right)$ & $50.8 \pm 2.3$ & $51.1_{-2.4}^{+2.9}$ & $86.4 \pm 1.4$ & $76.1_{-2.9}^{+0.9}$ \\
\hline$K_{2}\left(\mathrm{~km} \mathrm{~s}^{-1}\right)$ & $449_{-6}^{+1}$ & $487 \pm 3$ & $371 \pm 3$ & $444 \pm 4$ \\
\hline$i\left(^{\circ}\right)$ & $80.76 \pm 0.19$ & $79.94 \pm 0.19$ & $86.9_{-0.2}^{+0.5}$ & $83.29_{-0.10}^{+0.29}$ \\
\hline $\log g$ & $8.15_{-0.04}^{+0.01}$ & $8.63 \pm 0.03$ & $8.186 \pm 0.019$ & $8.77 \pm 0.04$ \\
\hline$T_{1}(\mathrm{~K})$ & $15500 \pm 1700$ & $14500 \pm 2200$ & $11000 \pm 1000$ & $17400 \pm 1900$ \\
\hline$d(\mathrm{pc})$ & $550 \pm 60$ & $430 \pm 60$ & $340 \pm 40$ & $380 \pm 40$ \\
\hline Parameter & GY Cne & IY UMa & OY Car & SDSS 0901 \\
\hline$q$ & $0.448_{-0.021}^{+0.014}$ & $0.146_{-0.001}^{+0.009}$ & $0.1065_{-0.0029}^{+0.0009}$ & $0.182_{-0.004}^{+0.009}$ \\
\hline$M_{1}\left(\mathrm{M}_{\odot}\right)$ & $0.881 \pm 0.016$ & $0.955_{-0.028}^{+0.013}$ & $0.882_{-0.015}^{+0.011}$ & $0.752_{-0.018}^{+0.024}$ \\
\hline$R_{1}\left(\mathrm{R}_{\odot}\right)$ & $0.00976_{-0.00018}^{+0.00021}$ & $0.0087_{-0.0001}^{+0.0003}$ & $0.00957_{-0.00012}^{+0.00018}$ & $0.01105_{-0.00029}^{+0.00022}$ \\
\hline$M_{2}\left(\mathrm{M}_{\odot}\right)$ & $0.394_{-0.022}^{+0.016}$ & $0.141 \pm 0.007$ & $0.093_{-0.001}^{+0.004}$ & $0.138 \pm 0.007$ \\
\hline$R_{2}\left(\mathrm{R}_{\odot}\right)$ & $0.446_{-0.009}^{+0.006}$ & $0.1770 \pm 0.0028$ & $0.1388_{-0.0003}^{+0.0018}$ & $0.182 \pm 0.003$ \\
\hline$a\left(\mathrm{R}_{\odot}\right)$ & $1.429 \pm 0.012$ & $0.765_{-0.009}^{+0.004}$ & $0.662 \pm 0.003$ & $0.739 \pm 0.007$ \\
\hline$K_{1}\left(\mathrm{~km} \mathrm{~s}^{-1}\right)$ & $125 \pm 4$ & $66_{-1}^{+4}$ & $50.4 \pm 0.9$ & $73 \pm 3$ \\
\hline$K_{2}\left(\mathrm{~km} \mathrm{~s}^{-1}\right)$ & $278.0 \pm 2.4$ & $453 \pm 3$ & $475.9 \pm 2.1$ & $401 \pm 3$ \\
\hline$i\left(^{\circ}\right)$ & $77.06_{-0.18}^{+0.29}$ & $84.9_{-0.5}^{+0.1}$ & $83.27_{-0.13}^{+0.10}$ & $81.4_{-0.3}^{+0.1}$ \\
\hline $\log g$ & $8.40 \pm 0.019$ & $8.54 \pm 0.03$ & $8.422_{-0.013}^{+0.017}$ & $8.228_{-0.025}^{+0.022}$ \\
\hline$T_{1}(\mathrm{~K})$ & $25900 \pm 2300$ & - & $18600_{-1600}^{+2800}$ & $14900 \pm 2000$ \\
\hline$d(\mathrm{pc})$ & $320 \pm 30$ & - & $90 \pm 5$ & $600 \pm 70$ \\
\hline Parameter & SDSS 1006 & SDSS 1152 & SDSS 1501 & SSS100615 \\
\hline$q$ & $0.46 \pm 0.03$ & $0.153_{-0.011}^{+0.015}$ & $0.084 \pm 0.004$ & $0.095 \pm 0.004$ \\
\hline$M_{1}\left(\mathrm{M}_{\odot}\right)$ & $0.82 \pm 0.11$ & $0.62 \pm 0.04$ & $0.723_{-0.013}^{+0.017}$ & $0.88 \pm 0.03$ \\
\hline$R_{1}\left(\mathrm{R}_{\odot}\right)$ & $0.0102 \pm 0.0013$ & $0.0129 \pm 0.0006$ & $0.01142_{-0.00022}^{+0.00016}$ & $0.0095 \pm 0.0003$ \\
\hline$M_{2}\left(\mathrm{M}_{\odot}\right)$ & $0.37 \pm 0.06$ & $0.094_{-0.009}^{+0.016}$ & $0.061 \pm 0.004$ & $0.083 \pm 0.005$ \\
\hline$R_{2}\left(\mathrm{R}_{\odot}\right)$ & $0.457_{-0.026}^{+0.022}$ & $0.147 \pm 0.006$ & $0.1129_{-0.0016}^{+0.0025}$ & $0.1276_{-0.0024}^{+0.0028}$ \\
\hline$a\left(\mathrm{R}_{\odot}\right)$ & $1.46 \pm 0.07$ & $0.627 \pm 0.014$ & $0.574 \pm 0.004$ & $0.628 \pm 0.007$ \\
\hline$K_{1}\left(\mathrm{~km} \mathrm{~s}^{-1}\right)$ & $124 \pm 9$ & $62 \pm 5$ & $39.5_{-1.3}^{+2.2}$ & $46.5_{-1.7}^{+2.2}$ \\
\hline$K_{2}\left(\mathrm{~km} \mathrm{~s}^{-1}\right)$ & $270 \pm 13$ & $402 \pm 7$ & $468 \pm 3$ & $493 \pm 5$ \\
\hline$i\left(^{\circ}\right)$ & $83.1_{-0.7}^{+1.2}$ & $82.6 \pm 0.5$ & $83.89_{-0.27}^{+0.20}$ & $85.1 \pm 0.3$ \\
\hline $\log g$ & $8.33 \pm 0.13$ & $8.01 \pm 0.05$ & $8.182_{-0.019}^{+0.016}$ & $8.43 \pm 0.03$ \\
\hline$T_{1}(\mathrm{~K})$ & - & $15900 \pm 2000$ & $\begin{array}{l}13400 \pm 1100(2004) \\
14900 \pm 1000(2012)\end{array}$ & $13600 \pm 1500$ \\
\hline$d(\mathrm{pc})$ & - & $610 \pm 80$ & $\begin{array}{l}400 \pm 30(2004) \\
338 \pm 21(2012)\end{array}$ & $350 \pm 30$ \\
\hline Parameter & SSS130413 & V713 Cep & Z Cha & \\
\hline$q$ & $0.169_{-0.006}^{+0.011}$ & $0.246_{-0.014}^{+0.006}$ & $0.189 \pm 0.004$ & \\
\hline$M_{1}\left(\mathrm{M}_{\odot}\right)$ & $0.84 \pm 0.03$ & $0.703_{-0.015}^{+0.012}$ & $0.803 \pm 0.014$ & \\
\hline$R_{1}\left(\mathrm{R}_{\odot}\right)$ & $0.0102_{-0.0002}^{+0.0006}$ & $0.01173_{-0.00015}^{+0.00020}$ & $0.01046 \pm 0.00017$ & \\
\hline$M_{2}\left(\mathrm{M}_{\odot}\right)$ & $0.140_{-0.008}^{+0.012}$ & $0.176_{-0.018}^{+0.007}$ & $0.152 \pm 0.005$ & \\
\hline$R_{2}\left(\mathrm{R}_{\odot}\right)$ & $0.163 \pm 0.004$ & $0.208_{-0.005}^{+0.002}$ & $0.1820 \pm 0.0020$ & \\
\hline$a\left(\mathrm{R}_{\odot}\right)$ & $0.680_{-0.011}^{+0.007}$ & $0.781 \pm 0.006$ & $0.734 \pm 0.005$ & \\
\hline$K_{1}\left(\mathrm{~km} \mathrm{~s}^{-1}\right)$ & $75 \pm 4$ & $91_{-5}^{+2}$ & $78.4_{-1.8}^{+1.4}$ & \\
\hline$K_{2}\left(\mathrm{~km} \mathrm{~s}^{-1}\right)$ & $443_{-7}^{+3}$ & $367.6_{-2.3}^{+2.6}$ & $413.2_{-2.0}^{+2.5}$ & \\
\hline$i\left(^{\circ}\right)$ & $82.5 \pm 0.3$ & $81.7 \pm 0.3$ & $80.44 \pm 0.11$ & \\
\hline $\log g$ & $8.35 \pm 0.04$ & $8.147_{-0.014}^{+0.017}$ & $8.304 \pm 0.016$ & \\
\hline$T_{1}(\mathrm{~K})$ & $24000 \pm 3000$ & $17000_{-3000}^{+6000}$ & $16300 \pm 1400$ & \\
\hline$d(\mathrm{pc})$ & $240 \pm 40$ & $320 \pm 30$ & $103 \pm 6$ & \\
\hline
\end{tabular}



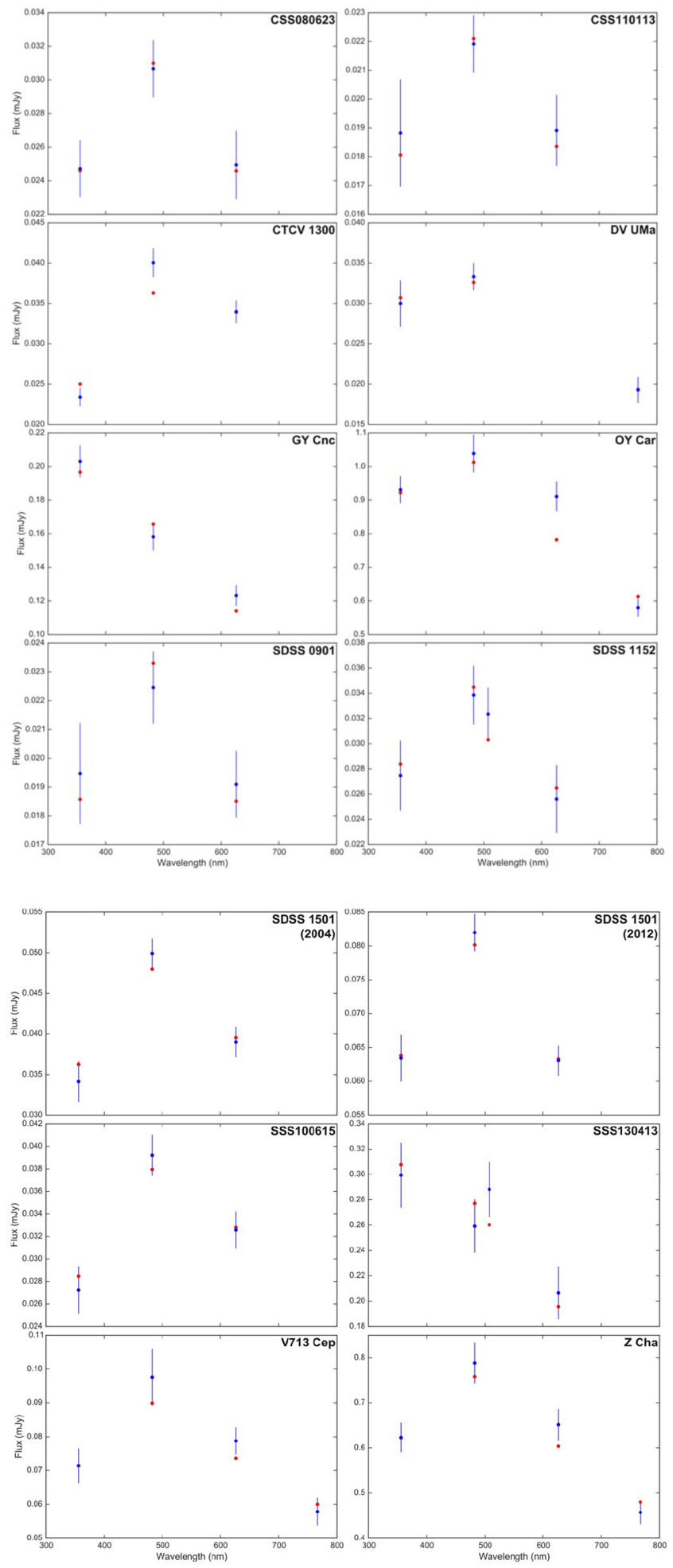

Figure 2. White dwarf fluxes for $13 \mathrm{CVs}$, showing the white dwarf fluxes from the eclipse model fits (blue) and white dwarf atmosphere predictions (red), at wavelengths corresponding to $u^{\prime}(355.7 \mathrm{~nm}), g^{\prime}(482.5 \mathrm{~nm}), K G 5$ $(507.5 \mathrm{~nm}), r^{\prime}(626.1 \mathrm{~nm})$, and $i^{\prime}(767.2 \mathrm{~nm})$ filters. The name of each system is displayed in the top-right corner of each plot.

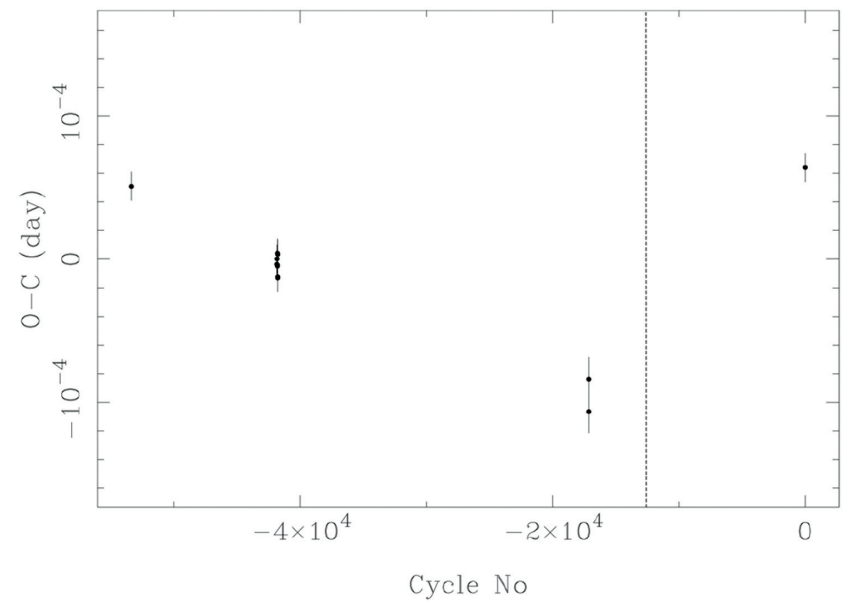

Figure 3. O-C diagram for all 12 available ULTRACAM eclipses of SDSS 1501 , spanning $\sim 8 \mathrm{yr}$. The vertical dashed line corresponds to $2010 \mathrm{Sep}$, when SDSS 1501 was reportedly observed in superoutburst. The $y$-axis covers $\pm 15 \mathrm{~s}$.

distance from Gaia DR2, including a weak distance prior (BailerJones et al. 2018). However, the most likely distance from Gaia DR2 is $340 \mathrm{pc}$; favouring the 2012 distance estimate. The white dwarf flux fitting was repeated for both eclipses, but this time with $d$ held fixed at $360 \mathrm{pc}$. This now gives $T_{1}(2004)=12100 \pm 300 \mathrm{~K}$ and $T_{1}(2012)=15800 \pm 300 \mathrm{~K}$, a much larger increase of $3700 \mathrm{~K}$.

Such a large discrepancy in $T_{1}$ indicates that the white dwarf in SDSS 1501 underwent a period of enhanced accretion between 2004 and 2012, most likely a superoutburst. According to vsnet-alert $12169,{ }^{3}$ the superoutburst occurred in 2010 Sep, with the observer claiming to have observed SDSS 1501 in outburst in addition to obtaining part of a superhump. Unfortunately, there is not enough coverage of this outburst to determine a superhump period.

In addition to the white dwarf flux variations, SDSS 1501 also exhibits small orbital period variations. The white dwarf-dominated SDSS 1501 eclipses enable very precise mid-eclipse times to be obtained. We show the mid-eclipse times - after the subtraction of a linear ephemeris - in Fig. 3. The orbital period of SDSS 1501 appears to depart from linearity by approximately $\pm 7 \mathrm{~s}$ over the $\sim 8$ yr ULTRACAM observational baseline. Such variations are not uncommon in $\mathrm{CV}$ s, and are thought to be caused by a magnetically driven process within the donor. However, they are not observed in CVs with donors of spectral type later than M6 (Bours et al. 2016), due to magnetic activity in the donor decreasing with later spectral types. SDSS 1501's donor mass obtained through eclipse modelling is substellar $\left(0.061 \pm 0.004 \mathrm{M}_{\odot}\right)$, strongly indicating a spectral type later than M6, and so the observation of period variations is surprising.

A logical deduction from looking at Fig. 3 is that the superoutburst from 2010 Sep (dashed line) may have caused the observed change in orbital period, as the ephemeris appears approximately linear up until this point. In this scenario, the 2012 eclipse occurs $\sim 21 \mathrm{~s}$ later than expected, implying an increase in SDSS 1501's orbital period of $0.0016 \mathrm{~s}\left(\Delta P_{\text {orb }} / P_{\text {orb }}=3.2 \times 10^{-7}\right)$. It is not clear how the superoutburst could have caused such a large change in the orbital period. If some fraction of the disc mass was ejected during superoutburst, we would expect $\Delta P_{\text {orb }} / P_{\text {orb }}=2 M_{\mathrm{ej}} /\left(M_{1}+M_{2}\right)$, where $M_{\mathrm{ej}}$ is the mass ejected. This implies ejected masses of 


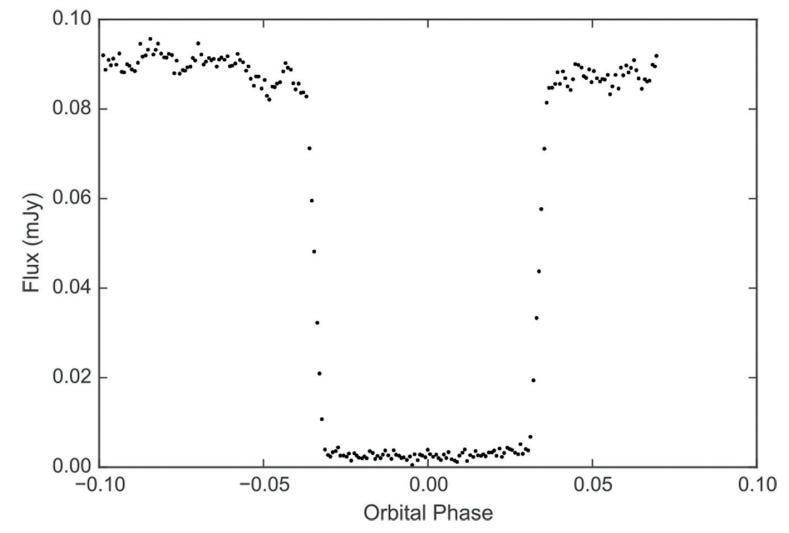

Figure 4. $g^{\prime}$-band eclipse light curve (2015 June 24, cycle no. 11955) of V713 Cep during a low state.

$10^{-7} \mathrm{M}_{\odot}$, and disc masses in excess of this. A period change might be induced by a change in the quadropole moment of the white dwarf and disc, due to the disc draining on to the white dwarf. In this case, Applegate (1992) gives

$\Delta P_{\mathrm{orb}} / P_{\mathrm{orb}} \approx-\frac{9 \Delta Q}{M_{1} a^{2}}$,

where $\Delta Q$ is the change in quadropole moment. We can obtain an order-of-magnitude estimate for $\Delta Q$ if we approximate the disc as a ring of mass $M_{\mathrm{d}}$ and radius $a / 3$, and assume that during superoutburst the disc completely drains on to the white dwarf, giving $\Delta Q \approx$ $-M_{\mathrm{d}} a^{2} / 9$. Therefore $\Delta P_{\text {orb }} / P_{\text {orb }} \approx M_{\mathrm{d}} / M_{1}$, again implying disc masses of order $10^{-7} \mathrm{M}_{\odot}$. With SDSS 1501's system parameters known (Table 2), the pre-outburst white dwarf temperature can be used to determine a medium-term average mass transfer rate for SDSS 1501 (Townsley \& Bildsten 2003, 2004; Townsley \& Gänsicke 2009) of $\dot{M}=9.3 \times 10^{-11} \mathrm{M}_{\odot} \mathrm{yr}^{-1}$. Period minimum systems are observed to have superoutburst cycles of order 20 $30 \mathrm{yr}$. Therefore the required disc masses are unrealistic, and the 2010 Sep superoutburst is not (at least not fully) responsible for the period variations exhibited by SDSS 1501. Another possible cause of the period variations is the presence of a third body within the system; however, additional precise mid-eclipse timings are required in order to investigate this further.

\subsection{Observed low state of V713 Cep}

The ULTRACAM/ULTRASPEC data archive contain a total of 15 V713 Cep eclipses, with two ULTRACAM eclipses (cycle nos. 11 $\left[u^{\prime} g^{\prime} r^{\prime}\right]$ and $\left.3655\left[u^{\prime} g^{\prime} i^{\prime}\right]\right)$ showing clear bright-spot features suitable for eclipse modelling. A feature of these two eclipses is a notable disc contribution (see Fig. A14), which is seen in all other V713 Cep eclipses in the archive, with the exception of one. The ULTRACAM $u^{\prime} g^{\prime} r^{\prime}$ eclipse of 2015 Jun 24 (cycle no. 11955, $g^{\prime}$-band eclipse shown in Fig. 4) contains no obvious signs of either a disc or bright-spot eclipse, and at first glance resembles an eclipse of a detached, nonaccreting binary. However, on closer inspection there are signs of flickering outside of white dwarf eclipse, as well as a very slight curvature inside eclipse. These two features are both evidence for the presence of an - albeit considerably diminished - accretion disc. A dwindling accretion disc and no sign of a bright-spot indicates that the secondary has stopped supplying the disc with material and the system is in what is known as a 'low state'.
Low states are relatively common phenomena for both magnetic CVs and a subgroup of novalike (NL) CVs called VY Scl stars; however, they appear to be very rare (and unexpected) for $\mathrm{DNe}$ below the period gap. In fact, there is only one other documented occurrence in the literature - an extended ( $>2 \mathrm{yr}$ ) low state of IR Com (Manser \& Gänsicke 2014). Given the rarity of low states in DNe, it is notable that IR Com and V713 Cep have similar orbital periods, just at the lower edge of the period gap. With only one eclipse of V713 Cep obtained during its low state, it is not known exactly how long this low state lasted. An upper limit of $403 \mathrm{~d}$ can be estimated based on the timings of other ULTRACAM eclipses, and therefore it was significantly shorter than the low state of IR Com.

\section{DISCUSSION}

With the new and revised system parameters obtained in this work, we now discuss what impact these results may have on the current understanding of CVs and their evolution. In what follows, we combine the parameters presented here with a compilation of reliable parameters for $46 \mathrm{CVs}$ from the literature. This compilation is presented in Table B1 (Supporting Information).

It has been shown that there is a significant discrepancy between the mean white dwarf mass in the field and that within CVs. Zorotovic et al. (2011) obtained a mean CV white dwarf mass of $0.82 \pm 0.03 \mathrm{M}_{\odot}$, and an intrinsic scatter of white dwarf masses of $\sigma=0.15 \mathrm{M}_{\odot}$. With the updated sample of $\mathrm{CV}$ masses now available, we can revise the mean white dwarf mass in CVs, following the procedure outlined in appendix B of Knigge (2006), to $0.81 \pm 0.02 \mathrm{M}_{\odot}\left(\sigma=0.13 \mathrm{M}_{\odot}\right)$, entirely consistent with Zorotovic et al's value.

One way to explain the presence of high white dwarf masses in $\mathrm{CVs}$ is through white dwarf mass growth through steady accretion across the lifetime of a CV. Since CVs evolve to shorter orbital periods over their lives, this requires the observation of higher white dwarf masses in systems with lower orbital periods. To test this, $\left\langle M_{1}\right\rangle$ was re-calculated for 31 systems below the period gap $\left(P_{\text {orb }} \sim 2.15 \mathrm{hrs}\right)$, giving $\left\langle M_{1}\right\rangle$ (below gap) $=0.81 \pm 0.02 \mathrm{M}_{\odot}$ ( $\left.\sigma=0.10 \mathrm{M}_{\odot}\right)$, and for 16 systems above the gap $\left(P_{\text {orb }} \sim 3.18 \mathrm{hrs}\right)$, giving $\left\langle M_{1}\right\rangle$ (below gap $)=0.82 \pm 0.02 \mathrm{M}_{\odot} \quad\left(\sigma=0.10 \mathrm{M}_{\odot}\right)$. We therefore see no evidence for white dwarf mass growth in CVs. While white dwarf mass growth in CVs appears doubtful, further precise white dwarf masses from systems at long period $(>3 \mathrm{~h})$ are required before it can be entirely dismissed.

\subsection{Testing the validity of the empirical CAML model}

An alternative explanation for the high white dwarf mass in CVs was proposed by Schreiber et al. (2016). The authors put forward an empirical consequential angular momentum loss (eCAML) model, which produces a dynamical stability limit on $q$, causing systems with low-mass white dwarfs to become unstable to mass transfer. These systems consequently merge, removing them from the CV population. The eCAML model is attractive as it can simultaneously explain the low observed space density of CVs (Belloni et al. 2018) and the origin of isolated low-mass white dwarfs (Zorotovic \& Schreiber 2017).

The top-left plot of fig. 2 in Schreiber et al. (2016) was updated to take into account the results of this work (Fig. 5). This plot is in $M_{2}$ versus $q$ parameter space, with regions (grey) that are theoretically prohibited due to constraints put on $M_{1}$. The dark grey prohibited region in the bottom right of Fig. 5 is an upper mass 


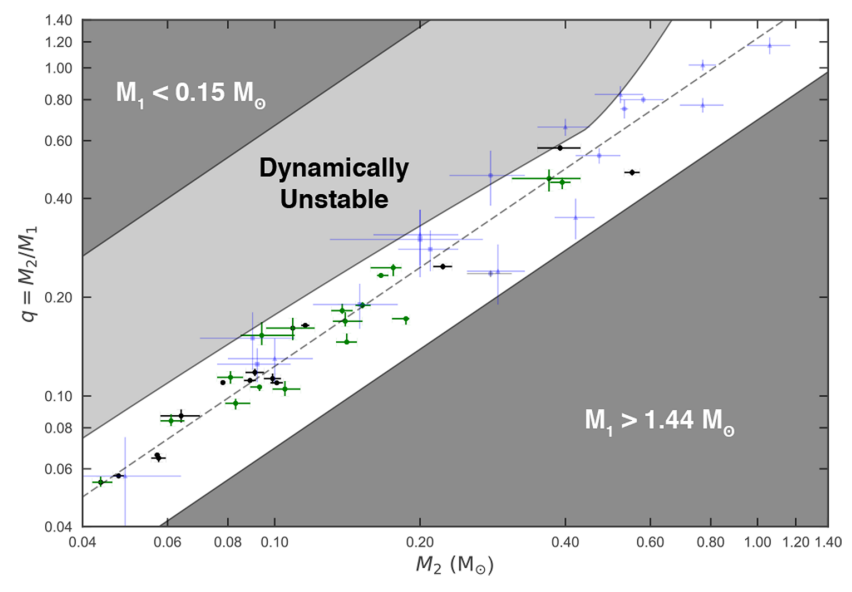

Figure 5. $q$ versus $M_{2}$ plot for CVs. The grey regions are theoretically prohibited due to constraints put on $M_{1}$. The dark grey regions cover unrealistically low white dwarf masses $\left(\lesssim 0.15 \mathrm{M}_{\odot}\right)$ and masses greater than the Chandrasekhar mass limit $\left(1.44 \mathrm{M}_{\odot}\right)$, while the light grey region is forbidden by the empirical consequential angular momentum loss (eCAML) model of Schreiber et al. (2016). The dashed grey line represents the mean value of $M_{1}$ from this work. The green and black points represent masses obtained from eclipse modelling of ULTRACAM/ULTRASPEC data, either from this work (green) or otherwise (black). The faint blue points represent measured CV masses from other methods: eclipse modelling of other data (circles), contact phase timing (squares), and radial velocity (triangles).

limit on $M_{1}$, resulting from the Chandrasekhar mass limit of a white dwarf $\left(1.44 \mathrm{M}_{\odot}\right)$. The light grey prohibited region is a lower mass limit on $M_{1}$ and is a consequence of the dynamical stability limit on $q$ supplied by the eCAML model. Also plotted in Fig. 5 are systems with measured $M_{2}$ and $q$, either from this work (green points) or elsewhere (black/blue points; see Table B1, Supporting Information). These systems with measured system parameters provide a test of the eCAML model, as all should lie within the valid region (white). Any systems lying inside the prohibited dynamically unstable region would compromise the credibility of the model.

All systems modelled in this work lie comfortably within the valid region of Fig. 5, along with the vast majority of other systems. Two appear to (just) violate the dynamical instability constraint, namely SDSS 0756+0858 (Tovmassian et al. 2014) and DQ Her (Horne, Welsh \& Wade 1993); however, both systems could feasibly be stable under the eCAML model after taking into account their uncertainties. This outcome offers support to the validity of the eCAML model as a solution to the CV white dwarf mass problem; however, a much larger sample of systems with precise system parameters is necessary in order to provide a more stringent test of the model.

\subsection{Reviewing the properties of the period spike}

The period spike is a feature of the orbital period distribution which is expected to occur as systems 'pile-up' near the orbital period minimum due to the long evolutionary time-scale. It was finally observed by Gänsicke et al. (2009) through analysing the orbital period distribution of newly identified CVs from SDSS (York et al. 2000). These systems were all identified spectroscopically (e.g. Szkody et al. 2002), and therefore not affected by the same biases/limitations as systems discovered through other means, e.g. DN outbursts and X-ray emission (see Gänsicke et al. 2009 for more details). Spectroscopic identification, coupled with a survey depth of $g^{\prime} \sim 19.5$, gives this particular sample the ability to provide the

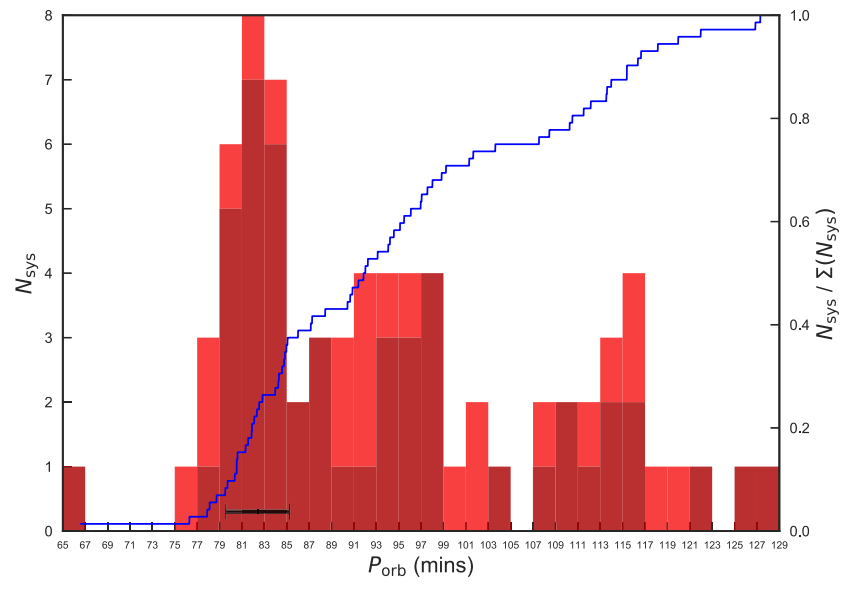

Figure 6. Histogram (red) and cumulative plot (blue) for 72 spectroscopically identified (from SDSS and 6dFGS) CVs below the period gap with precise $P_{\text {orb }}$ measurements (sub-30 s errors). For comparison, the sample of Gänsicke et al. (2009) is also shown (dark red histogram), in addition to the position and FWHM of the period spike estimated in the same study (black bar).

closest representation of the true orbital period distribution of $\mathrm{CVs}$ to date, a claim supported by the emergence of the long predictedbut-elusive period spike at the period minimum. Gänsicke et al. (2009) produced estimates for the location $(82.4 \pm 0.7 \mathrm{~min})$ and width [full width at half-maximum $(\mathrm{FWHM}=5.7 \mathrm{~min})$ ] of the period spike. Eight years on, the sample has increased and more $P_{\text {orb }}$ measurements have become available, enabling the orbital period distribution - and in particular the properties of the period spike to be reviewed.

The Gänsicke et al. (2009) sample consisted of 49 spectroscopically identified SDSS CVs below the period gap $\left(P_{\text {orb }} \lesssim\right.$ 129 min; Knigge 2006) with precise $P_{\text {orb }}$ measurements (errors $<30$ s). Precise $P_{\text {orb }}$ measurements for an additional 23 systems (and updated measurements for a handful from the original sample) have since become available, increasing the sample to 72 systems. Of the new systems, six are eclipsing systems with observations using ULTRACAM/ULTRASPEC, 10 are from Thorstensen et al. (2015, 2017), and the remaining seven are from the Ritter \& Kolb (2003) catalogue (v7.24; see references within). All systems were discovered by the SDSS (e.g. Szkody et al. 2011) except two, PHL 1445 and CSS110113, which were discovered by the 6dF Galaxy Survey (6dFGS; Jones et al. 2004).

Fig. 6 shows the orbital period distribution of all 72 spectroscopically identified CVs in the form of both a histogram (red) and cumulative plot (blue). As with the Gänsicke et al. (2009) sample (dark red histogram), the new sample shows a clear accumulation of systems centred around $\sim 82 \mathrm{~min}$, which is clearly identifiable as the period spike. Estimating $P_{\text {spike }}$ involved the fitting of a Gaussian distribution to the orbital period distribution between 77 and $87 \mathrm{~min}$. An estimate of $P_{\text {spike }}=82.7 \pm 0.4 \mathrm{~min}(\sigma=2.35 \mathrm{~min}$, FWHM $=5.53 \mathrm{~min}$ ) was obtained, which is largely unchanged from the Gänsicke et al. (2009) sample. This is not surprising, as the majority ( $\sim 75$ per cent) of additional systems have $P_{\text {orb }}>89 \mathrm{~min}$, and therefore do not belong to the period spike.

We note here that there is a hint of bi-modality in the period distribution of systems below the period gap, with a dearth of systems with orbital periods around 88 min. A Hartigan dip test (Hartigan \& Hartigan 1985) reveals that this is not statistically significant. 
Table 3. Orbital $\left(P_{\mathrm{orb}}\right)$ and superhump $\left(P_{\mathrm{sh}}\right)$ periods of the systems used to calibrate the $\epsilon(q)$ relation. The majority of systems are SU UMa-type DNe; however, the bottom four are CNe/NLs. $P_{\mathrm{sh}}^{\mathrm{B}}$ and $P_{\mathrm{sh}}^{\mathrm{C}}$ are the periods for stage $\mathrm{B}$ and $\mathrm{C}$ superhumps, respectively. See Tables 2 and $\mathrm{B} 1$ for $q$ values.

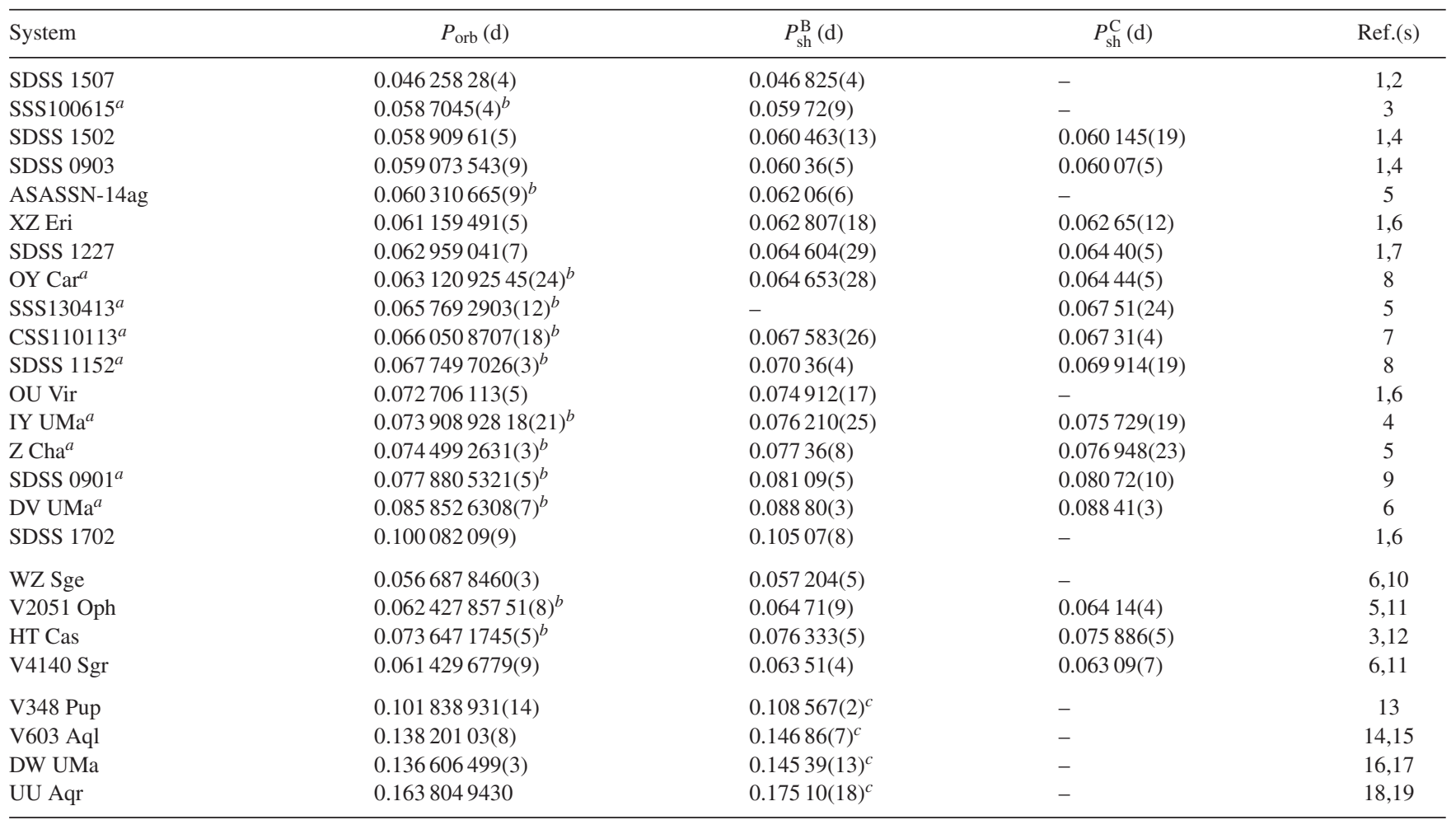

Notes: References: (1) Savoury et al. (2011), (2) Patterson et al. (2017), (3) Kato et al. (2016), (4) Kato et al. (2010), (5) Kato et al. (2015), (6) Kato et al. (2009), (7) Kato et al. (2012), (8) Kato et al. (2017), (9) Kato et al. (2013), (10) Patterson (1998), (11) Baptista et al. (2003), (12) Horne, Wood \& Stiening (1991), (13) Rolfe, Haswell \& Patterson (2000), (14) Peters \& Thorstensen (2006), (15) Patterson et al. (1997), (16) Araujo-Betancor et al. (2003), (17) Patterson et al. (2002), (18) Baptista \& Bortoletto (2008), and (19) Patterson et al. (2005).

${ }^{a}$ Updated $q$ value produced in this work (Table 2).

${ }^{b} P_{\text {orb }}$ from this work.

${ }^{c}$ Superhump period from permanent superhumps.

\subsection{Updating the calibration of the superhump period excess-mass ratio relation}

During superoutburst the accretion disc is driven into an elliptical state by resonances between the donor star and material within the disc. Tidal interactions between the elliptical disc and the donor lead to periodic fluctuations in the elliptical, precessing, disc known as superhumps. The disc precesses at a slow rate, with a period $\left(P_{\text {prec }}\right)$ significantly longer than $P_{\text {orb }}$. These two periods therefore both contribute to the formation of the superhump period $\left(P_{\mathrm{sh}}\right)$, which is simply the 'beat period' of $P_{\text {prec }}$ and $P_{\text {orb }}$ (Hellier 2001):

$\frac{1}{P_{\mathrm{sh}}}=\frac{1}{P_{\mathrm{orb}}}-\frac{1}{P_{\mathrm{prec}}}$.

$P_{\text {sh }}$ is therefore usually a few per cent longer than $P_{\text {orb }}$, but does not stay constant throughout the superoutburst. In fact, a superoutburst can be split up into three distinct stages (A, B, and C), with sharp transitions observed between each stage. Stage A represents the start of the superoutburst, with a long, stable $P_{\text {sh }}$. Stage B is the middle part of the superoutburst, with a shorter, unstable $P_{\mathrm{sh}}$. The final stage (C) exhibits the shortest $P_{\mathrm{sh}}$, which is stable once again (Olech et al. 2003; Kato et al. 2009). The general trend of decreasing $P_{\text {sh }}$ across the superoutburst hints at an increasing $P_{\text {prec }}$ (from equation 1 ) and therefore a dwindling disc radius (Murray 2000).

The superhump excess $(\epsilon)$ is defined as $\epsilon=\frac{P_{\text {sh }}-P_{\text {orb }}}{P_{\text {orb }}}$, and is directly related to the mass ratio, $q$. A calibration of this relationship (e.g. Patterson et al. 2005; Knigge 2006) allows estimates of mass ratios for all superhumping systems. From this current work and the work of others (e.g. Savoury et al. 2011), new potential calibration systems have emerged, in addition to revised $q$ values for existing calibration systems. Revised superhump periods have also been measured, courtesy of the SU UMa-type DNe survey of Kato et al. (2009, 2010, 2012, 2013, 2014a,b, 2015, 2016, 2017). With all of these new measurements becoming available since the work of Knigge (2006), it is appropriate to update the calibration of the $\epsilon(q)$ relation.

Table 3 contains all of the calibrating systems currently available, ${ }^{4}$ along with their orbital and superhump periods (and references). The two superhump period columns, $P_{\mathrm{sh}}^{\mathrm{B}}$ and $P_{\mathrm{sh}}^{\mathrm{C}}$, represent the superhump periods during stage $B$ and stage $C$ of superoutburst, respectively. All but the final four systems in Table 3 are SU UMa-type DNe that undergo superoutbursts. The other four systems are either Classical Novae or Novalikes that display permanent superhumps, and it is assumed these superhump periods resemble those of $P_{\mathrm{sh}}^{\mathrm{B}}$ for SU UMa-type DNe.

For each system in Table 3 , the superhump period excess was calculated for stage $\mathrm{B}\left(\epsilon_{\mathrm{B}}\right)$ and stage $\mathrm{C}\left(\epsilon_{\mathrm{C}}\right)$ depending on $P_{\mathrm{sh}}^{\mathrm{B}} / P_{\mathrm{sh}}^{\mathrm{C}}$ availability. Fig. 7 shows $\epsilon_{\mathrm{B}}$ plotted against $q$ for the 24

\footnotetext{
${ }^{4}$ The calibration system KV UMa used by Knigge (2006) was not included on the basis of it being a low-mass X-ray binary, rather than a CV.
} 

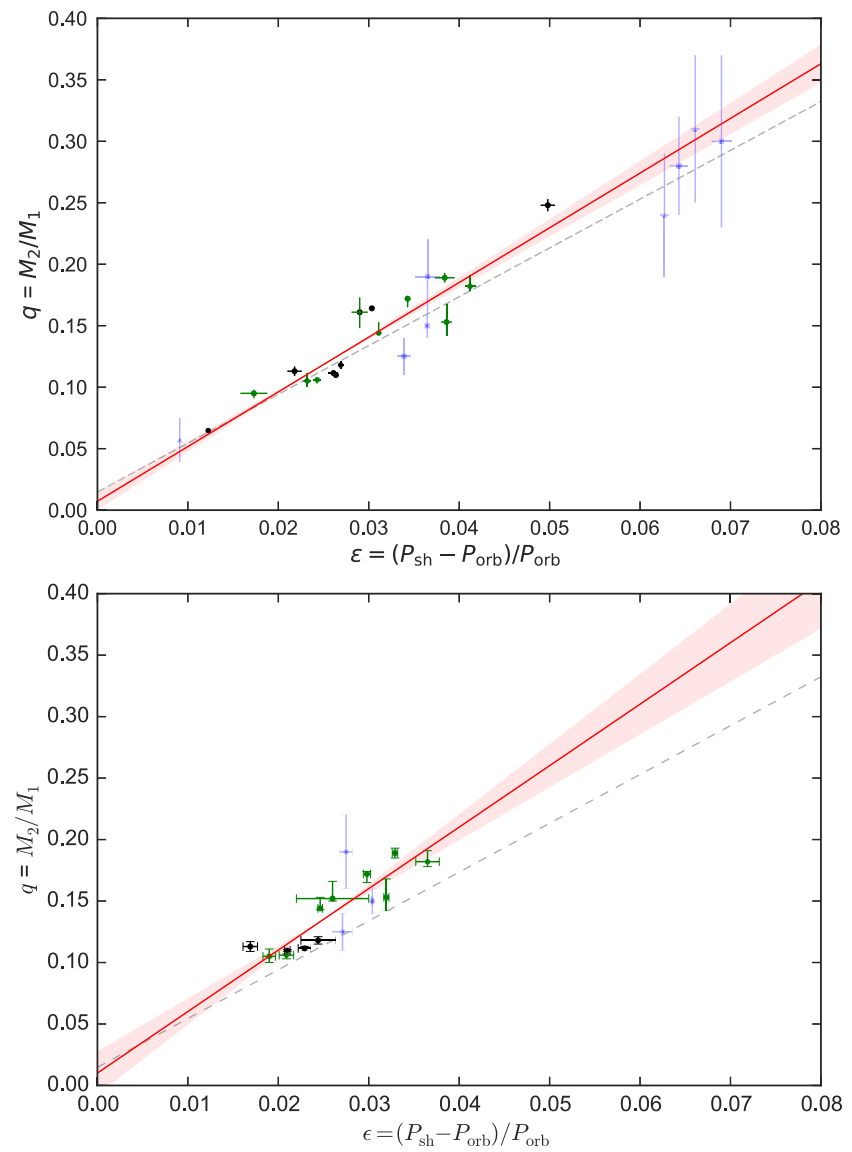

Figure 7. Measured $\epsilon_{\mathrm{B}}$ and $q$ values of superhumping and eclipsing CVs, with the same data point colour/shape scheme as Fig. 5. The dashed grey line shows the existing linear calibration of the $\epsilon(q)$ relation for superhumping CVs from Knigge (2006), while the red line shows an updated calibration from this work. The red shaded region represents $1 \sigma$ errors. The top plot shows the relationship for stage B superhumps, the bottom plot that for stage C superhumps.

calibration systems from Table 3 with available $P_{\mathrm{sh}}^{\mathrm{B}}$ measurements. The dashed grey line shows the existing calibration from Knigge (2006), while the red line represents the following, updated linear calibration:

$q\left(\epsilon_{\mathrm{B}}\right)=(0.118 \pm 0.003)+(4.45 \pm 0.28) \times\left(\epsilon_{\mathrm{B}}-0.025\right)$.

This updated calibration was obtained through the same $\chi^{2}$ minimization technique employed by Knigge (2006) (see appendix A of reference), and has an intrinsic dispersion $(\sigma)$ of 0.012 . While there is good coverage for systems with $0.1<q<0.2$, more calibration systems with $q$ outside this range are required in order to further constrain the gradient. For example, due to its position in Fig. 7, SDSS $1702(q \approx 0.25)$ has a rather large influence on the gradient, so therefore more systems with precisely measured values of $q$ greater than 0.2 are highly coveted. Unfortunately, this includes period gap systems, which are rare, and systems above the gap, for which precise measurements of $q$ are hard to obtain. It is clear from Fig. 7 that the new calibration has a steeper gradient that the existing one from Knigge (2006). A possible reason for this is the variation in measurement of $P_{\mathrm{sh}}$ between Patterson et al. (2005) and Kato et al. (2009); the sources of $P_{\text {sh }}$ for both the existing and new calibration, respectively. Patterson et al. (2005) measures $P_{\text {sh }}$ from 'common' superhumps, which typically cover stage B, but can also cover only a fraction of this stage or spread into stages A and $\mathrm{C}$.

The same treatment was given to the 15 calibration systems in Table 3 with available $P_{\mathrm{sh}}^{\mathrm{C}}$ measurements, producing the following linear relation (with $\sigma=0.012$ again inferred):

$q\left(\epsilon_{\mathrm{C}}\right)=(0.135 \pm 0.004)+(5.0 \pm 0.7) \times\left(\epsilon_{\mathrm{C}}-0.025\right)$.

This relation is also shown in Fig. 7.

\subsection{Donor masses and radii of superhumping CVs}

Given our updating of the superhump-mass ratio relations above, we revisit the analysis of donor star properties in Knigge (2006) and Knigge et al. (2011). First, $P_{\mathrm{sh}}$ values for all SU UMa-type $\mathrm{DNe}$ in the Patterson et al. (2005) sample (70 systems) were replaced by $P_{\mathrm{sh}}^{\mathrm{B}}$ measurements from the SU UMa-type DNe survey of Kato et al. (2009, 2010, 2012, 2013, 2014a,b, 2015, 2016, 2017). For a number of systems, $P_{\text {orb }}$ was also updated, either from measurements made by Kato et al. or additional studies (see references within Kato et al.). Values of $\epsilon_{\mathrm{B}}$ were obtained from $P_{\mathrm{sh}}^{\mathrm{B}}$ and $P_{\text {orb }}$, then subsequently converted into $q$ via the newly calibrated $\epsilon_{\mathrm{B}}(q)$ relation (equation 2). Equation (2) was also used to determine $q$ for the eight systems displaying permanent superhumps. Assuming a constant white dwarf mass of $\left\langle M_{1}\right\rangle=0.81 \mathrm{M}_{\odot}$, donor mass estimates were obtained for all systems in the superhumper sample.

As the donor fills its Roche lobe, the Eggleton (1983) approximation for the volume-averaged Roche lobe size, combined with Kepler's third law, can be used to obtain estimates for donor radii from $q, M_{2}$, and $P_{\text {orb }}$ :

$$
\frac{R_{2}}{\mathrm{R}_{\odot}}=0.2478\left(\frac{M_{2}}{\mathrm{M}_{\odot}}\right)^{1 / 3} P_{\mathrm{orb}}^{2 / 3}\left[\frac{q^{1 / 3}(1+q)^{1 / 3}}{0.6 q^{2 / 3}+\ln \left(1+q^{1 / 3}\right)}\right],
$$

where $P_{\text {orb }}$ is in units of h. The Eggleton (1983) approximation for the volume-averaged Roche lobe size is the same one used to determine $R_{2}$ for systems that have been eclipse modelled, establishing consistency between the superhumping and eclipsing samples. It is important to note that Knigge et al. (2011) use a more complex, accurate approximation for the volume-averaged size of the Roche lobe based on the results of Sirotkin \& Kim (2009), which represents the donor as a polytrope, rather than a point source. However, the advantage of using the Sirotkin \& Kim (2009) approximation is small, with only a $\sim 1$ per cent difference between the two approximations (fig. 3 of Knigge et al. 2011).

In addition to the 78 superhumper sample from Patterson et al. (2005), Kato et al. provide $P_{\mathrm{sh}}^{\mathrm{B}, \mathrm{C}}$ and $P_{\text {orb }}$ values for a further 147 systems. These systems were given the same treatment as the Patterson et al. (2005) sample (outlined above). A handful of systems only have available $P_{\mathrm{sh}}^{\mathrm{C}}$ values, in which case equation (3) was used. This brings the total number of superhumping systems with inferred donor properties to 225 .

\subsection{Updating the semi-empirical mass-radius relation for $\mathrm{CV}$ donor stars}

With donor masses and radii for 15 eclipsing systems in this work, a further 31 (mostly) eclipsing systems from the literature (see Table B1, Supporting Information) and 225 superhumpers, it is possible to update the mass-radius relation for $\mathrm{CV}$ donor stars from Knigge (2006) and Knigge et al. (2011). The same fitting 


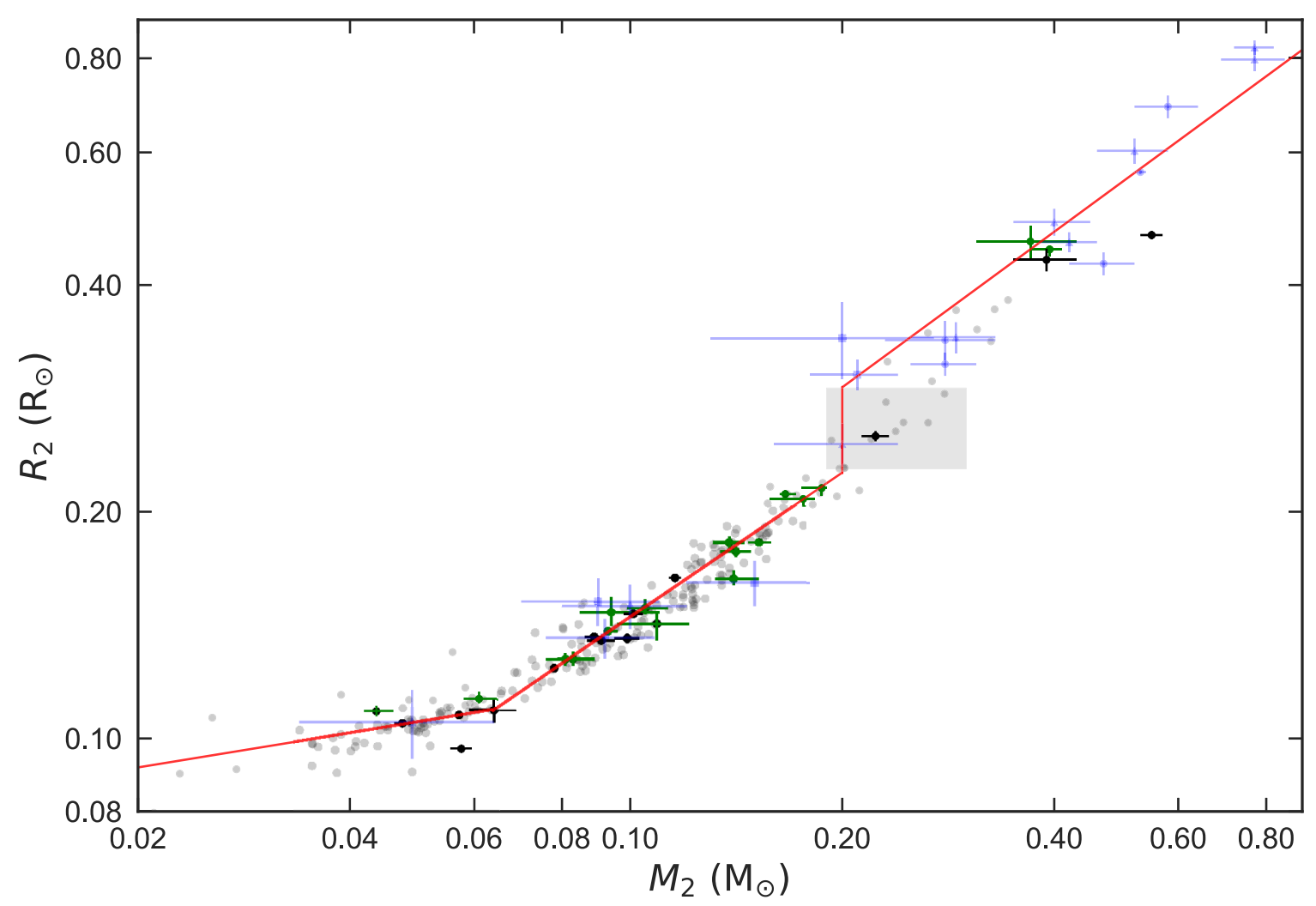

Figure 8. Measured CV donor masses $\left(M_{2}\right)$ and radii $\left(R_{2}\right)$. The data point colour/shape scheme is the same as in Fig. 5, but with additional superhumping systems (grey points), for which error bars have been omitted for clarity. The red line is the semi-empirical mass-radius relation from this work. The grey shaded region contains systems assumed to lie within the period gap, and is therefore not included in the updated broken-power-law fit.

procedure used by Knigge (2006) was followed to update the massradius relation. Assumptions for some parameters in this model are required, since they are not well-constrained by the donor masses and radii. Assumptions for the donor mass within the period gap $\left(M_{\text {conv }}\right)$, and the upper and lower $\left(P_{\text {gap, }+}, P_{\text {gap },-}\right)$ bounds of the period gap from Knigge et al. (2011) remained unchanged. We do adopt a smaller value for $P_{\text {bounce }}$ (called $P_{\min }$ in Knigge et al. 2011). $P_{\text {bounce }}$ is the orbital period where the pre-bounce and post-bounce power-law relationships intersect. Knigge et al. (2011) used the location of the period spike from Gänsicke et al. (2009) for $P_{\text {bounce }}$ However, real systems do not reach this orbital period, because the smooth track followed by real systems near period minimum is not well represented by two power laws. PHL 1445 (McAllister et al. 2015 ) is expected to be close to the absolute minimum period for main-sequence CVs, and so its orbital period of $76.3 \mathrm{~min}$ is used for $P_{\text {bounce }}$ here. The value of $M_{\text {bounce }}$ shown above was determined from the optimal short-period fit.

$$
\begin{array}{ll}
M_{\text {bounce }}=0.063_{-0.002}^{+0.005} \mathrm{M}_{\odot}, & P_{\text {bounce }}=76.3 \pm 1.0 \mathrm{~min} \\
M_{\text {conv }}=0.20 \pm 0.02 \mathrm{M}_{\odot}, & P_{\text {gap },-}=2.15 \pm 0.03 \mathrm{hrs}, \\
M_{\text {evol }} \simeq 0.6-0.8 \mathrm{M}_{\odot}, & P_{\text {gap },+}=3.18 \pm 0.04 \mathrm{hrs} .
\end{array}
$$

The donor masses and radii for all but 12 systems were included in the fits. The majority of these systems were excluded due to being period gap systems (see grey box in bottom plot of Fig. 8), while SDSS 1507 (outlying black data point in period bouncer regime) was excluded as it is known to be a Galactic halo object (Patterson, Thorstensen \& Knigge 2008; Uthas et al. 2011). The results from the three power-law fits are shown in Fig. 8, and take the following form:

$$
\frac{R_{2}}{\mathrm{R}_{\odot}}= \begin{cases}0.109 \pm 0.003\left(\frac{M_{2}}{M_{\text {bounce }}}\right)^{0.152 \pm 0.018} & M_{2}<M_{\text {bounce }} \\ 0.225 \pm 0.008\left(\frac{M_{2}}{M_{\text {conv }}}\right)^{0.636 \pm 0.012} & M_{\text {bounce }}<M_{2}<M_{\text {conv }} \\ 0.293 \pm 0.010\left(\frac{M_{2}}{M_{\text {conv }}}\right)^{0.69 \pm 0.05} & M_{\text {conv }}<M_{2}<M_{\text {evol }} .\end{cases}
$$

Comparing these results with Knigge et al. (2011), there is little change in the exponents of the mass-radius relation in both the long- and short-period regimes. One notable difference, however, is the amount of intrinsic scatter, $\sigma_{\text {int }}$, required for the short-period systems, reduced from approximately 0.02 to $0.005 \mathrm{R}_{\odot}$. The small scatter provides strong evidence for a very tight evolutionary path followed by non-evolved CV donors, implying little spread in AML loss rates for $\mathrm{CVs}$ with the same component masses. The scatter within the long-period regime, at $0.04 \mathrm{R}_{\odot}$, is almost a factor of 10 larger than that at short periods. Fig. 8 shows two outlying longperiod systems with $R_{2} \simeq 0.40 \mathrm{R}_{\odot}$, namely IP Peg (Copperwheat et al. 2010) and HS 0220+0603 (Rodríguez-Gil et al. 2015). The donors within these two systems are undersized for their masses, and may even be in thermal equilibrium, which is unexpected for a CV donor. It is possible that both IP Peg and HS 0220+0603 have donors in thermal equilibrium due to recently starting mass transfer.

The mass-radius relation for period-bouncers has changed significantly. The new power-law exponent of $0.152 \pm 0.018$ is much smaller than that of Knigge et al. (2011), a consequence of using lower values for both $M_{\text {bounce }}$ and $P_{\text {bounce }}$, in addition to the inclusion of many more period-bouncers in the new donor sample, which 


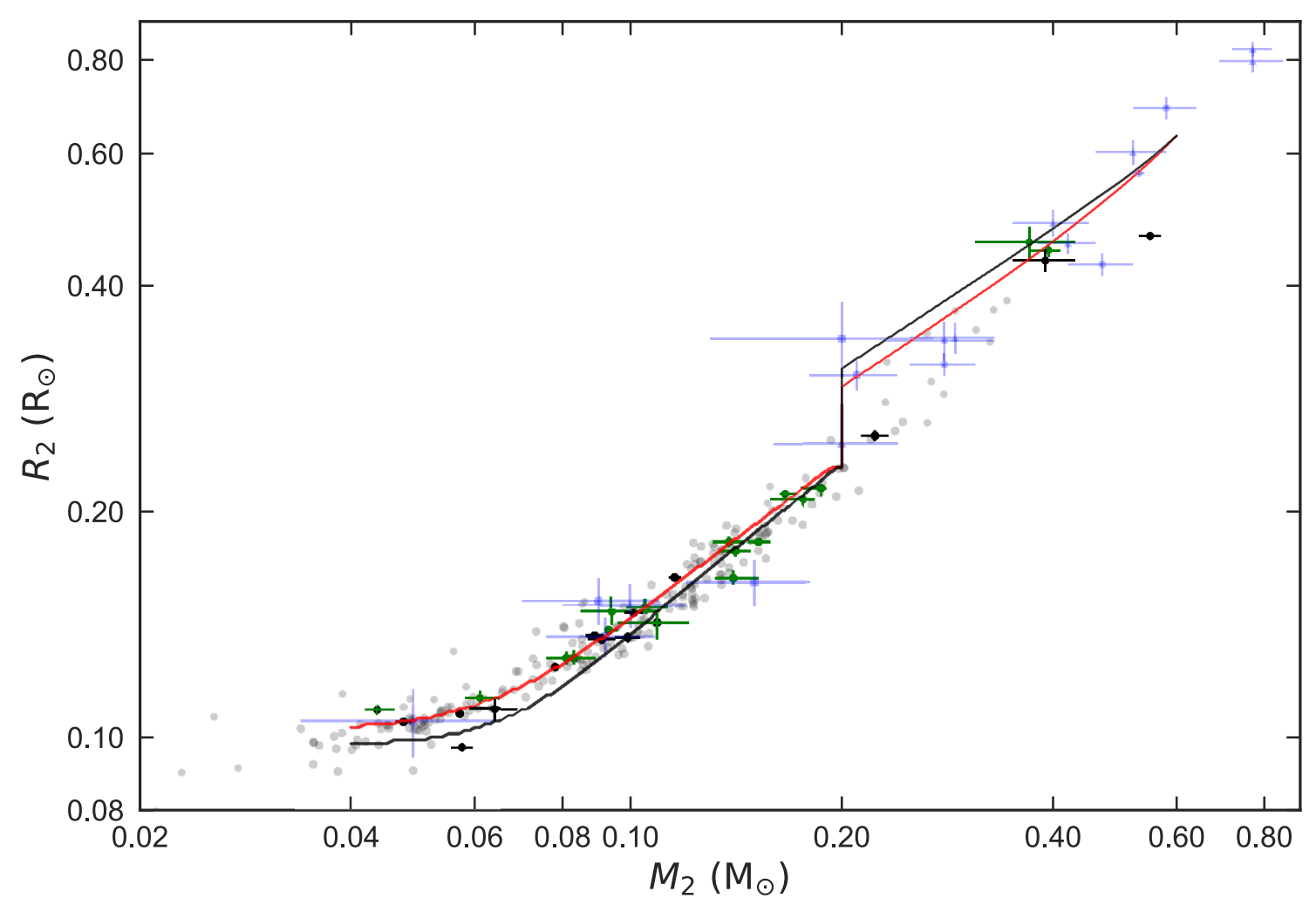

Figure 9. Measured CV donor masses $\left(M_{2}\right)$ and radii $\left(R_{2}\right)$. The data point colour/shape scheme is the same as in Fig. 8. The red and black lines represent the best fit $\left(f_{\mathrm{GR}}=2.47 \pm 0.22, f_{\mathrm{MB}}=0.66 \pm 0.05\right)$ and 'standard' ( $\left.f_{\mathrm{GR}}=f_{\mathrm{MB}}=1\right)$ evolutionary tracks from Knigge et al. (2011), respectively.

enables a better constraint of the power law in this regime. There has been a long-standing issue with the number of confirmed periodbounce CVs, which has always been much lower than the predicted 40-70 per cent (Kolb 1993; Goliasch \& Nelson 2015). Whilst the sample of donor masses collected here is far from homogeneous, and the presence of large numbers of superhumping systems introduces complicated selection effects, we note here that 30 per cent of our sample has a donor mass below $0.063 \mathrm{M}_{\odot}$ and are therefore likely to be period-bouncers.

\subsection{Comparison to theoretical CV evolution tracks}

In addition to a broken-power-law mass-radius relation for $\mathrm{CV}$ donors, Knigge et al. (2011) present a theoretical evolutionary track, produced with the aim of quantifying the secular mass transfer rate in CVs. The track which best reproduces their donor sample requires reduced magnetic braking above the gap $\left(f_{\mathrm{MB}}=0.66 \pm 0.05\right)$, but additional AML below the gap $\left(f_{\mathrm{GR}}=2.47 \pm 0.22\right)$. The donor sample presented in this work is shown in the $M_{2}-R_{2}$ and $P_{\text {orb }}-M_{2}$ planes in Figs 9 and 10, respectively. Also shown is the 'bestfitting' track from Knigge et al. (2011), and the 'standard' track $\left(f_{\mathrm{GR}}=f_{\mathrm{MB}}=1\right)$. It is clear from these figures that the best-fitting evolutionary track from Knigge et al. (2011) under predicts the donor mass at orbital periods below the period gap, and has a period minimum that is longer than that observed. This again implies that less additional AML is needed below the period gap than suggested by Knigge et al. (2011). In contrast, we find that the 'standard' track provides a better fit to the donor sample immediately below the gap, where the donor mass is in the range $0.10-0.20 \mathrm{M}_{\odot}$. This is most apparent in Fig. 10. Although the standard track is a good fit to systems immediately below the gap, it diverges from the donor sequence at lower masses, and predicts a period minimum shorter than the observed value. Therefore, the donor properties in $\mathrm{CVs}$ appear to argue for an additional source of AML that is small compared to gravitational radiation just below the period gap, but becomes more significant at shorter orbital periods and/or donor masses.

The eCAML model of Schreiber et al. (2016) might provide something similar to the behaviour required. All models of $\mathrm{CV}$ evolution require a term $v$, which expresses the AML which arises as a consequence of mass transfer. In the standard model, it is assumed that the mass lost from the white dwarf during nova eruptions carries with it the specific angular momentum of the white dwarf, leading to $v=M_{2}^{2} /\left(M_{1} M\right)$, where $M$ is the total mass of the system. In the eCAML model, an alternative form of $v \sim 0.35 / M_{1}$ is proposed. We used equation (1) from Knigge et al. (2011) to roughly estimate the mass-loss rates under the eCAML model at key points in the evolution of the donor. Just below the period gap, we take $M_{1}=$ $0.82 \mathrm{M}_{\odot}, M_{2}=0.15 \mathrm{M}_{\odot}$ and we assume the donor is roughly in thermal equilibrium, so the mass-radius index is $\xi=0.8$. This implies that in the eCAML model, mass-loss rates just after the period gap are only around 35 per cent higher than the 'standard', $f_{\mathrm{GR}}=1$, model. For systems near the period minimum, we take $M_{1}$ $=0.82 \mathrm{M}_{\odot}, M_{2}=0.065 \mathrm{M}_{\odot}$, and $\xi=-1 / 3$, which suggests massloss rates around 9 times higher than the $f_{\mathrm{GR}}=1$ case. Therefore, the eCAML model provides a mass-loss law which is qualitatively similar to the one implied by $\mathrm{CV}$ donor properties. However, it is worth bearing in mind that the $v \sim 0.35 / M_{1}$ prescription is not physically motivated. Schreiber et al. (2016) suggest that AML during nova outbursts might produce a similar behaviour, but the frequency of nova outbursts will drop as the accretion rate falls. Therefore, eCAML may be less important for CVs near the period 


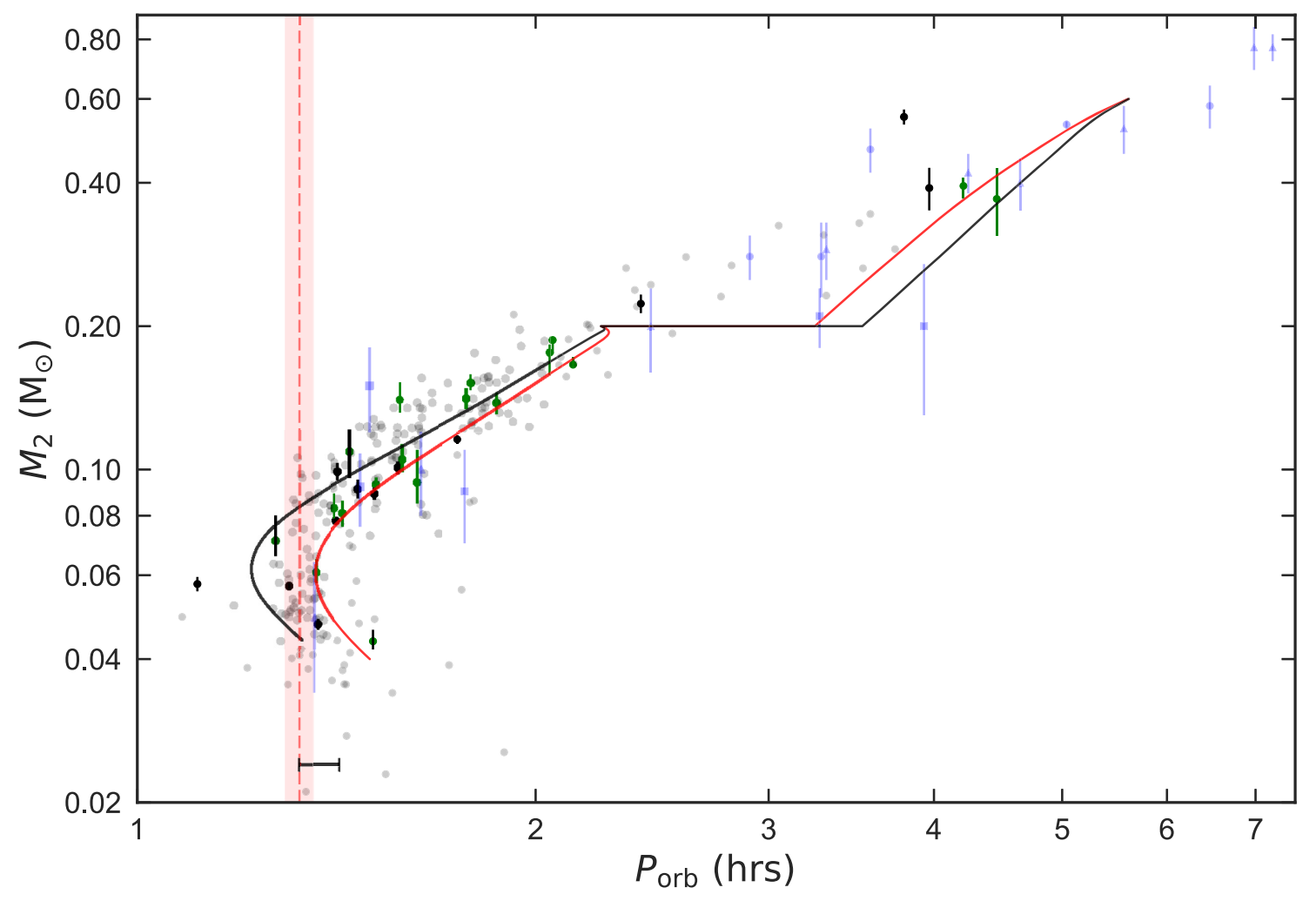

Figure 10. Measured CV donor masses $\left(M_{2}\right)$ as a function of orbital period $\left(P_{\text {orb }}\right)$. The data point colour/shape scheme is the same as in Fig. 8. The red and black lines represent the best fit $\left(f_{\mathrm{GR}}=2.47 \pm 0.22, f_{\mathrm{MB}}=0.66 \pm 0.05\right)$ and 'standard' $\left(f_{\mathrm{GR}}=f_{\mathrm{MB}}=1\right)$ evolutionary tracks from Knigge et al. (2011), respectively. The vertical dashed red line and shaded region is an estimate of the true $P_{\min }$ based on fitting a Gaussian distribution to the orbital periods in the range $76-82 \mathrm{~min}$.

minimum than implied above. It will require a physically plausible model of CV evolution, including AML during nova outbursts, to determine if such a model can reproduce both the highwhite dwarf mass in CVs and the $P_{\text {orb }}-M_{2}$ locus of the donor stars.

Finally, we note that our results introduce a tension between the donor masses and radii, and the temperatures of white dwarfs in CVs. As described in Townsley \& Gänsicke (2009), compressional heating of the white dwarfs due to accretion sets the equilibrium temperature of the white dwarf in a CV. The observed white dwarf temperature thus depends upon the accretion rate, averaged over the thermal time-scale of the non-degenerate layer on the white dwarf surface (Townsley \& Bildsten 2003). The best study of white dwarf temperatures in CVs to date is Pala et al. (2017), who show that the white dwarfs in CVs below the period gap imply AML rates approximately twice that implied by $f_{\mathrm{GR}}=1$. As discussed extensively in section 4 of Knigge et al. (2011), one plausible explanation for the discrepancy is the presence of mass transfer rate fluctuations, coupled with the fact that the white dwarf temperature reflects the mass transfer rate averaged over much shorter time-scales than the donor star radius. However, this would presumably lead to white dwarf temperatures scattered around the expected values; whereas they are systematically warmer than expected.

\subsection{The period minimum}

It is apparent from Fig. 10 that the current donor sample contains a sufficiently large number of systems at the shortest orbital periods to finally begin to reveal the locus of $\mathrm{CV}$ s evolving through the period minimum. The period minimum of the current donor sample covers an approximate period range of 76-82 $\mathrm{min}(1.27-1.37 \mathrm{~h})$. Fitting a Gaussian distribution to the donor sample within this period range returned the following estimates for both the period minimum $\left(P_{\min }=79.6 \pm 0.2 \mathrm{~min}\right)$ and its width $(\mathrm{FWHM}=4.0 \mathrm{~min})$. These estimates for $P_{\min }$ and its width are shown by the red vertical dashed line and shaded area within Fig. 10.

It was briefly mentioned in Section 5.5 that the observed location of the period minimum appears to be slightly lower than the value $P_{\min }=81.8 \pm 0.9$ min predicted by the best-fitting track of Knigge et al. (2011). The new measurement of $P_{\min }$ from the donor sample confirms this, with the two $P_{\min }$ estimates differing by approximately $2.4 \sigma$. A lower value of $P_{\min }$ than the existing estimate of Knigge et al. (2011) was previously hinted at by McAllister et al. (2015). Fig. 10 shows that with the new estimate for $P_{\min }$, PHL $1445\left(P_{\text {orb }}=1.27 \mathrm{~h}\right)$ and SDSS $1433\left(P_{\text {orb }}=1.30 \mathrm{~h}\right)$ are no longer troublesome outliers.

\section{CONCLUSIONS}

We present new measurements of the system parameters for 15 eclipsing CVs, six of which are published for the first time. We also compile a list of reliable system parameter determinations from the literature. We use these measurements to refine the calibration of the relationship between superhump period excess and mass ratio; allowing us to estimate the donor properties of $225 \mathrm{CV}$ s showing superhump phenomena. This provides an extensive sample of CVs with known system parameters which we can use to test models of CV evolution. 
We confirm the high average white dwarf mass in CVs, but we find no evidence for a trend in white dwarf mass with orbital period. Contrary to previous studies, we find that the donor properties of $\mathrm{CVs}$ immediately below the period gap are consistent with the standard model, in which AML due to magnetic braking is small compared to gravitational radiation. We do, however, find that CVs at shorter orbital periods and lower masses still require an additional source of AML. We argue that the eCAML model of Schreiber et al. (2016) predicts an AML law that is qualitatively similar to this behaviour. We find that, for systems below the period gap, donor radii at a given orbital period show a very small intrinsic scatter of only $0.005 \mathrm{R}_{\odot}$, suggesting that most CVs below the gap follow a common evolutionary path. We estimate a value for the orbital period minimum of $79.6 \pm 0.2 \mathrm{~min}$, shorter than previously estimated by Knigge et al. (2011).

The CVs with donor properties estimated from superhumps show a sizeable fraction of systems which appear to have evolved past the period minimum. As a result, 30 per cent of our sample appear to be post-period minimum systems. This hints that post-period minimum systems may be as common as models predict, but the superhump sample is strongly biased towards low-mass ratios. The advent of Gaia means that detailed follow up of a relatively complete volume-limited sample may resolve this question in the near future.

\section{ACKNOWLEDGEMENTS}

VSD, SPL, ULTRACAM and ULTRASPEC are supported by the Science and Technology Facilities Council grant ST/R000964/1. This work has made use of data obtained at the Thai National Observatory on Doi Inthanon, operated by NARIT. VSD, TRM and SPL acknowledge the support of the Royal Society and the Leverhulme Trust for the operation of ULTRASPEC at the TNT. The WHT is operated on the island of La Palma by the Isaac Newton Group of Telescopes in the Spanish Observatorio del Roque de los Muchachos of the Instituto de Astrofísica de Canarias. This work is based on observations collected at the European Organisation for Astronomical Research in the Southern Hemisphere.

\section{REFERENCES}

Applegate J. H., 1992, ApJ, 385, 621

Araujo-Betancor S. et al., 2003, ApJ, 583, 437

Arenas J., Catalán M. S., Augusteijn T., Retter A., 2000, MNRAS, 311, 135

Arnold S., Berg R. A., Duthie J. G., 1976, ApJ, 206, 790

Bailer-Jones C. A. L., Rybizki J., Fouesneau M., Mantelet G., Andrae R., 2018, AJ, 156, 58

Baptista R., Bortoletto A., 2008, ApJ, 676, 1240

Baptista R., Steiner J. E., Cieslinski D., 1994, ApJ, 433, 332

Baptista R., Borges B. W., Bond H. E., Jablonski F., Steiner J. E., Grauer A. D., 2003, MNRAS, 345, 889

Belloni D., Schreiber M. R., Zorotovic M., Iłkiewicz K., Hurley J. R., Giersz M., Lagos F., 2018, MNRAS, 478, 5626

Bergeron P., Wesemael F., Beauchamp A., 1995, PASP, 107, 1047

Borges B. W., Baptista R., 2005, A\&A, 437, 235

Bours M. C. P. et al., 2016, MNRAS, 460, 3873

Copperwheat C. M. et al., 2012, MNRAS, 421, 149

Copperwheat C. M., Marsh T. R., Dhillon V. S., Littlefair S. P., Hickman R., Gänsicke B. T., Southworth J., 2010, MNRAS, 402, 1824

Dhillon V. S. et al., 2007, MNRAS, 378, 825

Dhillon V. S. et al., 2014, MNRAS, 444, 4009

Dillon M. et al., 2008, MNRAS, 386, 1568

Echevarría J., de la Fuente E., Costero R., 2007, AJ, 134, 262

Echevarría J., Ramírez-Torres A., Michel R., Hernández Santisteban J. V., 2016, MNRAS, 461, 1576
Eggleton P. P., 1983, ApJ, 268, 368

Gänsicke B. T., Long K. S., Barstow M. A., Hubeny I., 2006, ApJ, 639, 1039

Gänsicke B. T. et al., 2009, MNRAS, 397, 2170

Goliasch J., Nelson L., 2015, ApJ, 809, 80

Hardy L. K. et al., 2017, MNRAS, 465, 4968

Hartigan J. A., Hartigan P., 1985, Ann. Stat., 13, 70

Hellier C., 2001, Cataclysmic Variable Stars: How and Why they Vary. Springer-Praxis, New York

Hernández Santisteban J. V., Knigge C., Pretorius M. L., Sullivan M., Warner B., 2018, MNRAS, 473, 3241

Hernandez M. S., Zharikov S., Neustroev V., Tovmassian G., 2017, MNRAS, 470, 1960

Horne K., Wood J. H., Stiening R. F., 1991, ApJ, 378, 271

Horne K., Welsh W. F., Wade R. A., 1993, ApJ, 410, 357

Howell S. B., Warnock A., Mason K. O., Reichert G. A., Kreidl T. J., 1988, MNRAS, 233, 79

Jones D. H. et al., 2004, MNRAS, 355, 747

Kato T. et al., 2009, PASJ, 61, S395

Kato T. et al., 2010, PASJ, 62, 1525

Kato T. et al., 2012, PASJ, 64, 21

Kato T. et al., 2013, PASJ, 65, 23

Kato T. et al., 2014a, PASJ, 66, 30

Kato T. et al., 2014b, PASJ, 66, 90

Kato T. et al., 2015, PASJ, 67, 105

Kato T. et al., 2016, PASJ, 68, 65

Kato T. et al., 2017, PASJ, 69, 75

Knigge C., 2006, MNRAS, 373, 484

Knigge C., Baraffe I., Patterson J., 2011, ApJS, 194, 28

Kolb U., 1993, A\&A, 271, 149

Lindegren L. et al., 2018, A\&A, 616, 2

Littlefair S. P., Dhillon V. S., Marsh T. R., Gänsicke B. T., Southworth J., Baraffe I., Watson C. A., Copperwheat C., 2008, MNRAS, 388, 1582

Littlefair S. P., Dhillon V. S., Gänsicke B. T., Bours M. C. P., Copperwheat C. M., Marsh T. R., 2014, MNRAS, 443, 718

Manser C. J., Gänsicke B. T., 2014, MNRAS, 442, L23

McAllister M. J. et al., 2015, MNRAS, 451, 114

McAllister M. J. et al., 2017a, MNRAS, 464, 1353

McAllister M. J. et al., 2017b, MNRAS, 467, 1024

Miszalski B. et al., 2016, MNRAS, 456, 633

Murray J. R., 2000, MNRAS, 314, L1

Nelemans G., Siess L., Repetto S., Toonen S., Phinney E. S., 2016, ApJ, 817, 69

Nogami D., Kato T., Baba H., Novák R., Lockley J. J., Somers M., 2001, MNRAS, 322, 79

Olech A., Schwarzenberg-Czerny A., Kedzierski P., Zloczewski K., Mularczyk K., Wisniewski M., 2003, AcA, 53, 175

Pala A. F. et al., 2017, MNRAS, 466, 2855

Patterson J. et al., 2000, PASP, 112, 1584

Patterson J. et al., 2002, PASP, 114, 1364

Patterson J. et al., 2005, PASP, 117, 1204

Patterson J. et al., 2017, Soc. Astron. Sci. Ann. Symp., 37, 1

Patterson J., 1998, PASP, 110, 1132

Patterson J., Kemp J., Saad J., Skillman D. R., Harvey D., Fried R., Thorstensen J. R., Ashley R., 1997, PASP, 109, 468

Patterson J., Thorstensen J. R., Knigge C., 2008, PASP, 120, 510

Peters C. S., Thorstensen J. R., 2006, PASP, 118, 687

Rappaport S., Joss P. C., Webbink R. F., 1982, ApJ, 254, 616

Rappaport S., Verbunt F., Joss P. C., 1983, ApJ, 275, 713

Ritter H., Kolb U., 2003, A\&A, 404, 301

Robinson E. L., 1974, ApJ, 193, 191

Rodríguez-Gil P. et al., 2015, MNRAS, 452, 146

Rodríguez-Gil P., Martínez-Pais I. G., Casares J., Villada M., van Zyl L., 2001, MNRAS, 328, 903

Rolfe D. J., Haswell C. A., Patterson J., 2000, MNRAS, 317, 759

Savoury C. D. J. et al., 2011, MNRAS, 415, 2025

Savoury C. D. J., Littlefair S. P., Marsh T. R., Dhillon V. S., Parsons S. G., Copperwheat C. M., Steeghs D., 2012, MNRAS, 422, 469 
Scaringi S., Groot P. J., Still M., 2013, MNRAS, 435, L68

Schreiber M. R., Zorotovic M., Wijnen T. P. G., 2016, MNRAS, 455, L16

Shafter A. W., Holland J. N., 2003, PASP, 115, 1105

Sing D. K., Green E. M., Howell S. B., Holberg J. B., Lopez-Morales M.,

Shaw J. S., Schmidt G. D., 2007, A\&A, 474, 951

Sirotkin F. V., Kim W.-T., 2009, ApJ, 698, 715

Smith J. A. et al., 2002, AJ, 123, 2121

Smith A. J., Haswell C. A., Hynes R. I., 2006, MNRAS, 369, 1537

Southworth J., Marsh T. R., Gänsicke B. T., Aungwerojwit A., Hakala P., de Martino D., Lehto H., 2007, MNRAS, 382, 1145

Southworth J., Hickman R. D. G., Marsh T. R., Rebassa-Mansergas A., Gänsicke B. T., Copperwheat C. M., Rodríguez-Gil P., 2009, A\&A, 507, 929

Southworth J., Copperwheat C. M., Gänsicke B. T., Pyrzas S., 2010, A\&A, $510, \mathrm{~A} 100$

Steeghs D., Marsh T., Knigge C., Maxted P. F. L., Kuulkers E., Skidmore W., 2001, ApJ, 562, L145

Steeghs D., Perryman M. A. C., Reynolds A., de Bruijne J. H. J., Marsh T., Dhillon V. S., Peacock A., 2003, MNRAS, 339, 810

Steeghs D., Howell S. B., Knigge C., Gänsicke B. T., Sion E. M., Welsh W. F., 2007, ApJ, 667, 442

Szkody P. et al., 2002, AJ, 123, 430

Szkody P. et al., 2011, AJ, 142, 181

Szkody P., Brownlee D. E., 1977, ApJ, 212, L113

Tappert C., Augusteijn T., Maza J., 2004, MNRAS, 354, 321

The Astropy Collaboration, 2018, AJ, 156, 123

Thoroughgood T. D. et al., 2005, MNRAS, 357, 881

Thoroughgood T. D., Dhillon V. S., Watson C. A., Buckley D. A. H., Steeghs D., Stevenson M. J., 2004, MNRAS, 353, 1135

Thorstensen J. R., 2000, PASP, 112, 1269

Thorstensen J. R., Taylor C. J., Peters C. S., Skinner J. N., Southworth J., Gänsicke B. T., 2015, AJ, 149, 128

Thorstensen J. R., Alper E. H., Weil K. E., 2016, AJ, 152, 226

Thorstensen J. R., Ringwald F. A., Taylor C. J., Sheets H. A., Peters C. S., Skinner J. N., Alper E. H., Weil K. E., 2017, Res. Notes Am. Astron. Soc., 1,29

Tovmassian G., Stephania Hernandez M., González-Buitrago D., Zharikov S., García-Díaz M. T., 2014, AJ, 147, 68
Townsley D. M., Bildsten L., 2003, ApJ, 596, L227

Townsley D. M., Bildsten L., 2004, ApJ, 600, 390

Townsley D. M., Gänsicke B. T., 2009, ApJ, 693, 1007

Tulloch S. M., Rodríguez-Gil P., Dhillon V. S., 2009, MNRAS, 397, L82

Uthas H., Knigge C., Long K. S., Patterson J., Thorstensen J., 2011, MNRAS, 414, L85

van Amerongen S., Damen E., Groot M., Kraakman H., van Paradijs J., 1987, MNRAS, 225, 93

Wade R. A., Horne K., 1988, ApJ, 324, 411

Welsh W. F., Froning C. S., Marsh T. R., Reimer T. W., Robinson E. L., Wood P. R., 2007, in Kang Y. W., Lee H.-W., Leung K.-C., Cheng K.-S., eds, ASP Conf. Ser. Vol. 362, The Seventh Pacific Rim Conference on Stellar Astrophysics. Astron. Soc. Pac., San Francisco, p. 241

Wood J., Horne K., Berriman G., Wade R., O’Donoghue D., Warner B., 1986, MNRAS, 219, 629

Wood J. H., Horne K., 1990, MNRAS, 242, 606

York D. G. et al., 2000, AJ, 120, 1579

Zorotovic M., Schreiber M. R., 2017, MNRAS, 466, L63

Zorotovic M., Schreiber M. R., Gänsicke B. T., 2011, A\&A, 536, A42

\section{SUPPORTING INFORMATION}

Supplementary data are available at MNRAS online.

Appendix B. Fits to eclipse light curves. Appendix C. Journal of observations.

Please note: Oxford University Press is not responsible for the content or functionality of any supporting materials supplied by the authors. Any queries (other than missing material) should be directed to the corresponding author for the article.

\section{APPENDIX A: SYSTEM PARAMETERS FOR SUPPLEMENTARY SYSTEMS}

The following table includes reliably determined system parameters for $\mathrm{CV}$ s from the literature.

Table A1. System parameters for supplementary systems included in Section 5 (Figs 5-10). The second-to-last column indicates the method used to obtain system parameters: EM - eclipse modelling ( $\mathrm{U}$ - using ULTRACAM/ULTRASPEC data), CPT - contact phase timing, RV - radial velocity, GR - gravitational redshift, SM - spectrophotometric modelling. For consistency, all $R_{2}$ values were calculated using equation 4 (ensuring all systems follow the same period-density relation). References: (1) Savoury et al. (2011), (2) McAllister et al. (2015), (3) McAllister et al. (2017a), (4) McAllister et al. (2017b) (5) Scaringi et al. (2013), (6) Littlefair et al. (2014), (7) Copperwheat et al. (2010), (8) Shafter \& Holland (2003), (9) Southworth et al. (2009), (10) Miszalski et al. (2016), (11) Rodríguez-Gil et al. (2015), (12) Sing et al. (2007), (13) Hernandez et al. (2017), (14) Tovmassian et al. (2014), (15) Steeghs et al. (2003), (16) Horne et al. (1991), (17) Wood \& Horne (1990), (18) Littlefair et al. (2008), (19) Baptista et al. (2003), (20) Borges \& Baptista (2005), (21) Araujo-Betancor et al. (2003), (22) Patterson et al. (2005), (23) Baptista \& Bortoletto (2008), (24) Baptista et al. (1994), (25) Thorstensen (2000), (26) Wade \& Horne (1988), (27) Echevarría et al. (2016), (28) Arnold et al. (1976), (29) Echevarría et al. (2007), (30) Horne et al. (1993), (31) Thoroughgood et al. (2005), (32) Rolfe et al. (2000), (33) Rodríguez-Gil et al. (2001), (34) Peters \& Thorstensen (2006), (35) Arenas et al. (2000), (36) Robinson (1974), (37) Welsh et al. (2007), (38) Thoroughgood et al. (2004), (39) Patterson (1998), (40) Steeghs et al. (2001), (41) Steeghs et al. (2007), (42) van Amerongen et al. (1987), (43) Smith et al. (2006), (44) Szkody \& Brownlee (1977), (45) Gänsicke et al. (2006).

\begin{tabular}{|c|c|c|c|c|c|c|c|}
\hline System & $\begin{array}{l}P_{\text {orb }} \\
\text { (d) }\end{array}$ & $q$ & $\begin{array}{c}M_{1} \\
\left(\mathrm{M}_{\odot}\right)\end{array}$ & $\begin{array}{c}M_{2} \\
\left(\mathrm{M}_{\odot}\right)\end{array}$ & $\begin{array}{c}R_{2} \\
\left(\mathrm{R}_{\odot}\right)\end{array}$ & Method & Ref. \\
\hline SDSS J1433+1011 & $0.054240679(2)$ & $0.0661(7)$ & $0.865(5)$ & $0.0571(7)$ & $0.1074(4)$ & $\mathrm{EM}(\mathrm{U})$ & 1 \\
\hline SDSS J1507+5230 & $0.04625828(4)$ & $0.0647(18)$ & $0.892(8)$ & $0.0575(20)$ & $0.0969(11)$ & $\mathrm{EM}(\mathrm{U})$ & 1 \\
\hline CTCV J2354 - 4700 & $0.065550270(1)$ & $0.1097(8)$ & $0.935(31)$ & $0.101(3)$ & $0.1463(16)$ & $\mathrm{EM}(\mathrm{U})$ & 1 \\
\hline SDSS J1152+4049* & $0.0677497026(3)^{\S}$ & $0.155(6)$ & $0.560(28)$ & $0.087(6)$ & $0.142(3)$ & $\operatorname{EM}(U)$ & 1 \\
\hline SDSS J0903+3300 & $0.059073543(9)$ & $0.113(4)$ & $0.872(11)$ & $0.099(4)$ & $0.1358(20)$ & $\mathrm{EM}(\mathrm{U})$ & 1 \\
\hline SDSS J1502+3334 & $0.05890961(5)$ & $0.1099(7)$ & $0.709(4)$ & $0.0781(8)$ & $0.1241(3)$ & $\mathrm{EM}(\mathrm{U})$ & 1 \\
\hline
\end{tabular}


Table A1 - continued

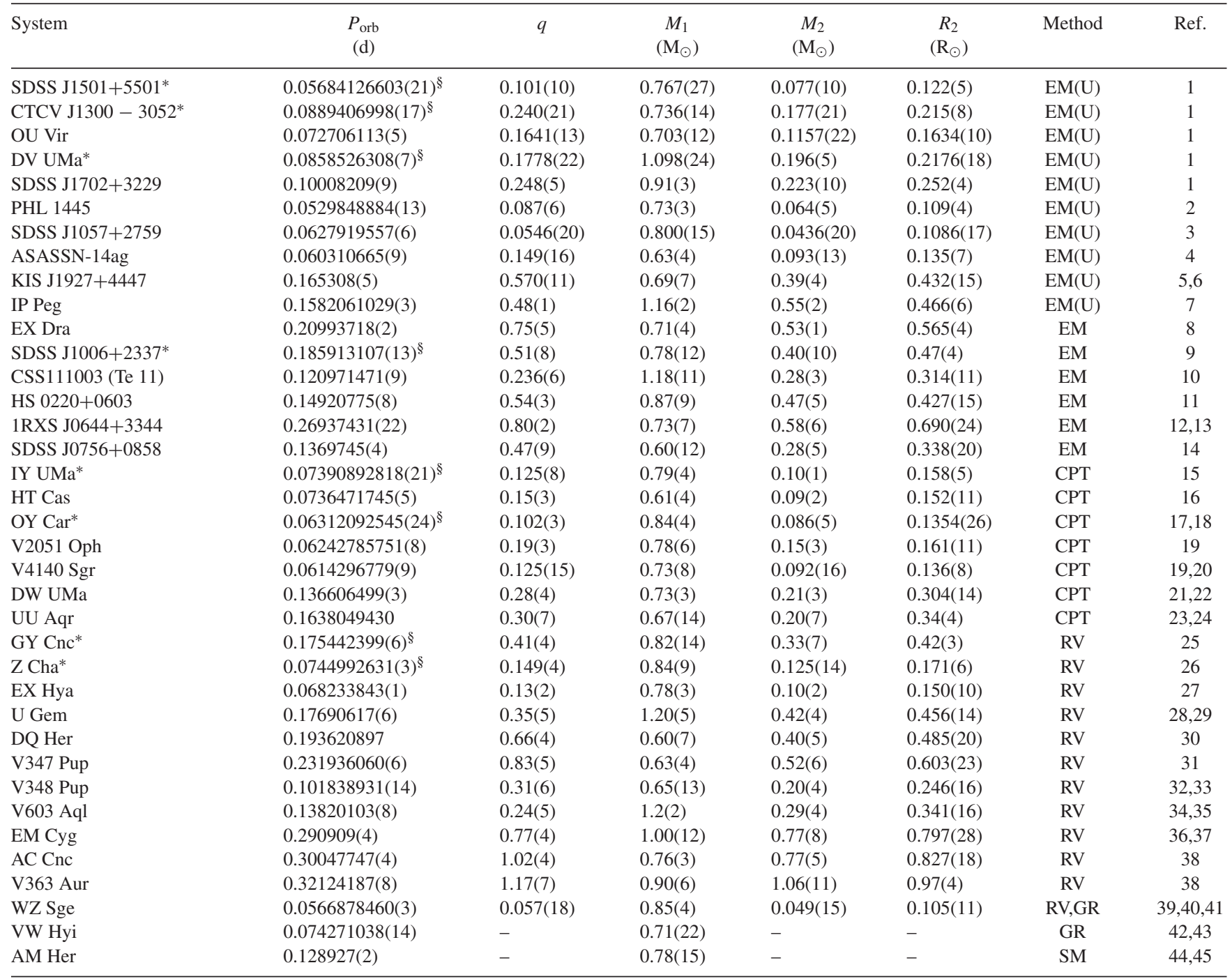

Note: ${ }^{*}$ Updated system parameters produced in this work (Table 2 ), ${ }^{\S} P_{\text {orb }}$ from this work

This paper has been typeset from a $\mathrm{T}_{\mathrm{E}} \mathrm{X} / \mathrm{L} \mathrm{T} \mathrm{E} \mathrm{X}$ file prepared by the author. 